\title{
Kernos
}

Revue internationale et pluridisciplinaire de religion grecque antique

12 | 1999

Varia

\section{La parenté légendaire à l'époque hellénistique}

Précisions méthodologiques

\section{Olivier Curty}

\section{Q OpenEdition}

1 Journals

\section{Édition électronique}

URL : http://journals.openedition.org/kernos/719

DOI : 10.4000/kernos.719

ISSN : 2034-7871

Éditeur

Centre international d'étude de la religion grecque antique

\section{Édition imprimée}

Date de publication : 1 janvier 1999

Pagination : 167-194

ISSN : 0776-3824

\section{Référence électronique}

Olivier Curty, "La parenté légendaire à l'époque hellénistique », Kernos [En ligne], 12 | 1999, mis en ligne le 13 avril 2011, consulté le 21 avril 2019. URL : http://journals.openedition.org/kernos/719 ; DOI :

10.4000/kernos.719 


\section{La parenté légendaire à l'êpoque hellénistique. Précisions méthodologiques}

\section{Réflexions générales sur la notion de parenté}

Ma thèse, publiée récemment ${ }^{1}$, a fait l'objet de plusieurs comptes rendus, certes divers dans leur ton, mais identiques dans leur rejet de mon interprétation du phénomène des relations entre cités en général et dans celle de la parenté en particulier ${ }^{2}$. L'objection principale est différente dans chaque article. Ainsi le premier auteur nie-t-il, entres autres, toute continuité entre les habitants de la période historique des cités grecques et leurs héros épichoriques de l'époque mythique ${ }^{3}$. Le second, décédé depuis lors, prétend qu'il faut toujours et systématiquement voir une différence de signification entre les mots syngeneia et oikeiotès ${ }^{4}$. Quant au troisième, il pense qu'il est illusoire d'établir une distinction entre, d'une part, les parentés légendaires, justifiées par des héros mythologiques et, d'autre part, les parentés de l'époque historique, issues des liens de colonisation. Ces deux sortes de parenté ne formeraient en réalité qu'un seul et même genre. En revanche, il faudrait différencier les parentés entre Grecs et Grecs, presque toujours réelles, de celles entre Grecs et non-Grecs qui sont « toujours et nécessairement légendaires $»^{5}$.

Les nombreuses différences d'analyse prouvent l'aspect fort discuté de la question et le caractère polémique tant du problème que de l'analyse qui en est tirée. Chacun a des idées très arrêtées sur le problème et, selon son tempérament, y accorde une importance plus ou moins grande dans l'élaboration des relations entre cités. C'est ainsi que tel esprit rationaliste tiendra

$1 \quad$ O. CuRty, Les parentés légendaires entre cités grecques: catalogue raisonné des

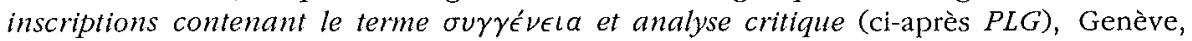
1995 (Hautes études du monde gréco-romain, 20).

2 Pour des comptes rendus ou citations plus favorables, voir p. ex., S. Hornblower [Dir.l, The Oxford Classical Dictionnary, Oxford, $1997^{3}$, s.v. kinship; Bull. Epigr., 109 (1996), $n^{\circ} 6$ (Ph. Gauthier) et Kemos, 11 (1998), p. 300 (A. Chaniotis).

3 J.M. HALL, CR, 47 (1997), p. 96-98.

4 E. WILL, Syngeneia, Oikeiotès, Philia, in $R P b$ (1995) [paru en 1997], p. 299-325.

5 A. Giovannini, Les relations de parenté entre cités grecques. À propos d'un livre récent, in $M H, 54$ (1997), p. 158-162. 
pour assuré que les parties en présence "n'y regarderont pas de si près » ${ }^{6}$ ou que tel autre écrira qu'« on pouvait bien invoquer celle-ci [i.e. la syngeneia] lorsqu'elle passait pour exister : cela ne faisait de mal à personne $»^{7}$, tandis que d'autres, plus scrupuleux, insisteront davantage sur le fait qu' « il ne faut pas les [i.e. les liens entre cités] traiter dans un esprit de frivolité dogmatique ${ }^{8}$. Cependant, malgré les différentes interprétations proposées et les avis divergents, je demeure convaincu de la justesse des miens.

Partons d'une constatation fort simple. La civilisation antique était pétrie de rhétorique. Ce qui signifie que, pour tous les actes de la vie, même et surtout les officiels, les Anciens recouraient au mythe pour justifier leur démarche et expliquer leur attitude. Ainsi, quand il s'agissait de nouer des relations entre cités, invoquaient-ils, chaque fois qu'ils le pouvaient, la parenté entre les deux cités, qui prenait sa source dans les mythes. Malheureusement, on ignore tout ou presque de la manière dont on en parlait et dont on justifiait ces parentés. Cependant, on a connaissance de tout un travail sur les mythes et sur leurs variantes locales qui a constitué une somme immense d'érudition. C'est dans celle-ci qu'on a inlassablement puisé les témoignages servant à établir des liens de parenté. On possède des traces de toute cette masse de travail par les inscriptions - essentiellement honorifiques - mentionnant les noms d'érudits qui ont travaillé sur ces matières. C'est ainsi, par exemple, que Démotélès d'Andros a rédigé un ouvrage, dans la première

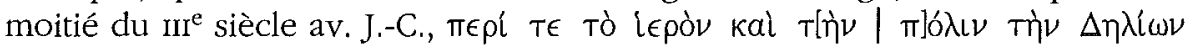

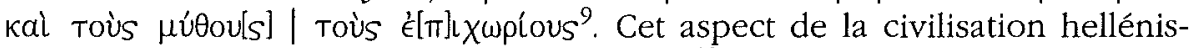
tique est fort connu et a déjà été bien étudié ${ }^{10}$. Il faut considérer, par conséquent, la manière dont les Grecs baignaient, dès leur enfance, dans un climat où la mythologie était systématiquement invoquée. Pour eux donc, la différence n'était pas, comme pour nous, entre, d'une part, une époque mythique irrationnelle et, d'autre part, une époque historique réelle. L'une et l'autre périodes coexistaient sans solution de continuité. Certes, la période mythique

6 M. Holleaux, Rome, la Grèce et les monarcbies bellénistiques, Paris, 1921, p. 5455, ก. 5 .

7 É. WiLL, art. cit. (n. 3), p. 320.

8 J. et L. Robert, Fouilles d'Amyzon en Carie, t. 1, Paris, 1983, p. 162, n. 31.

$9 \quad I G, \mathrm{XI}, 4,544$.

10 Voir, par exemple, l'étude de base de M. Guarducci, $M A L$, VI, 2 (1929), p. 629-665. Pour d'autres cas à ajouter à la liste dressée par M. Guarducci, J. et L. RoserT, Bull. Epigr. (1959), $\mathrm{n}^{\circ}$ 330. Voir également A. Chaniotis, Historie und Historiker in den griechischen Inscbriften, Stuttgart, 1988, p. 305-306 E 12 [n 76 PLG] et E 18. La vogue de ces recherches et le goût pour la mythologie locale se poursuivirent sous l'Empire comme l'attestent l'inscription trouvée à Labraunda, datée du $\mathrm{nI}^{\mathrm{e}}$ s. apr. J.-C. (J. Crampa, Labraunda. Swedish Excavations and Researcbes, vol. III, part 2: The Greek Inscriptions, Stockholm, 1972, $\mathrm{n}^{\circ}$ 66, p. 134-137), l'inscription de P. Anteius Antiochos d'Aigée, commentée de façon magistrale par L. Robert et reprise par A. Chaniotis, op. cit, p. 322-324 E 28 [n 5 PLG] ainsi que celle qui a été trouvée à Amphipolis, du III ${ }^{\mathrm{e}} \mathrm{s}$. apr. J.-C. (J. et L. Robert, Bull. Epigr. [1979], n² 271). 
contenait les exploits de héros et des faits que leur éloignement du présent avait assurément enjolivés, mais elle n'était pas fondamentalement différente de la période historique; avec ses héros et ses faits légendaires, elle permettait, d'une part, aux habitants des cités grecques de l'époque historique, de se construire tout un passé qu'ils pouvaient aisément invoquer selon leurs besoins. D'autre part, le traitement par les Anciens de ces récits légendaires et de ces traditions était fait de la même façon que de la matière historique. L'arbitrage rendu par Magnésie du Méandre, dans le dernier quart du II $^{\mathrm{e}}$ siècle av. J.-C., entre les cités crétoises d'Itanos et de Hiérapytna ${ }^{11}$, en apporte la confirmation. Avant de rendre leur sentence, en effet, les juges

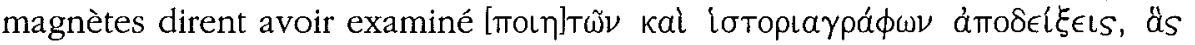

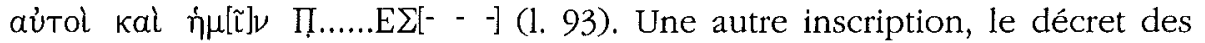
Épidamniens pour la reconnaissance du concours instauré par Magnésie du Méandre à la fin du $\mathrm{rrr}^{\mathrm{e}}$ siècle av. J.-C. en l'honneur d'Artémis Leucophryène, peut également être invoquée ${ }^{12}$. Les ambassadeurs magnètes, après s'être présentés devant le Conseil et l'Assemblée, ont montré à leurs auditeurs les

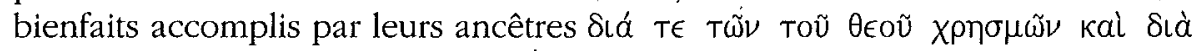

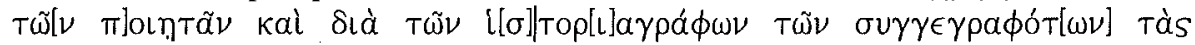

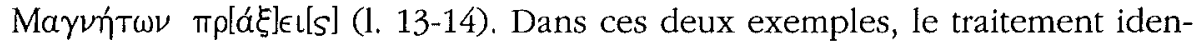
tique des poèmes, des sources proprement historiques (oracles) et des témoignages historiographiques prouve le caractère semblable que les Anciens attribuaient aux époques mythique et historique. Par conséquent, l'importance des récits légendaires dans les actes officiels est bien attestée. C'est aussi la raison pour laquelle la distinction établie par A. Giovannini entre les faits de la période mythique et ceux de l'époque historique ne me semble guère convaincante. L'importance de la question exige naturellement une réponse plus appropriée.

Avant de la donner, je voudrais d'abord analyser les différentes sortes de parenté qu'il faudrait envisager de reconnaître selon A. Giovannini. Pour lui, en effet, il faut distinguer les parentés entre Grecs et Grecs, de celles qui lient des Grecs à des non-Grecs. Dans cette optique, où classer précisément la cité carienne d'Alabanda qui, en 201/200 av. J.-C., était seulement réputée «parente » des Grecs, mais qui, au $\mathrm{uI}^{\mathrm{e}}$ siècle apr. J.-C., se proclama fièrement descendante lacédémonienne? La distinction, trop rigide, me semble inadéquate pour analyser le problème des liens entre cités. Si je résume brièvement la théorie d'A. Giovannini, la différence serait entre des parentés généalogiques, et par conséquent mythiques, liant surtout des Grecs à des nonGrecs tandis que les parentés issues de la colonisation, reflétant des liens réels, existeraient presque toujours entre Grecs et Grecs. "[Ces liens] sont réels dans le sens que les partenaires croient, peu importe que ce soit à tort ou à raison, qu'ils ont un passé historique commun ${ }^{13}$. Si nous reprenons

\footnotetext{
11 I. Creticae, III, IV, 9.

12 I. Magnesia, 46; Syll. ${ }^{3}, 560$; j'y reviendrai ensuite plus en détail.

13 A. Grovannini, art. cit. (n. 4), p. 162.
} 
l'exemple d'Alabanda, force est de constater que la réalité n'est pas aussi évidente que cette division tendrait à le faire croire. Certes, de prime abord, la distinction établie par A. Giovannini entre les liens unissant des Grecs à des non-Grecs «toujours et nécessairement légendaires ${ }^{14}$ et ceux que nouent des Grecs avec d'autres Grecs « presque toujours réels » ${ }^{15}$ semble confirmée. Quand Alabanda était une cité non grecque, ne se rattachait-elle pas aux Grecs par le biais de généalogies mythiques et lorsque la cité clama son origine lacédémonienne, ne put-elle pas revendiquer un œciste, confirmant de cette manière la réalité de son lien avec Sparte ? Cependant, il y eut une étape intermédiaire pendant laquelle Alabanda se rattacha à Carystos, cité d'Eubée, au moyen d'une généalogie mythique et non en vertu de quelque lien de colonisation. Pour expliquer ce fait, qui contredit la thèse d'A. Giovannini, on pourrait à la rigueur considérer ce cas comme s'ajoutant aux deux autres sans conteste d'origine mythique, c'est-à-dire à la parenté de Samè avec Magnésie du Méandre par l'intermédiaire de Magnès ${ }^{16}$, et à celle des Milésiens avec les Crétois par Apollon ${ }^{17}$. Nous aurions de cette manière trois attestations de liens remontant explicitement à la mythologie. D'un autre côté, il y a six $\operatorname{cas}^{18}$ de parenté explicitement justifiés par des rapports entre métropole et colonie. Ce sont Odessos et Tomis ${ }^{19}$, Istros et Apollonie Pontique $^{20}$, Cos et Camarina ${ }^{21}$, Cos et Géla ${ }^{22}$, Magnésie du Méandre et Antioche de Perside ${ }^{23}$, Magnésie du Méandre et Antioche de Pisidie ${ }^{24}$.

À côté de ces quelques cas que justifient soit la parenté mythique remontant par conséquent à l'époque légendaire soit les liens de colonisation qui appartiennent quant à eux à l'époque historique, la grande majorité des textes attestant une parenté entre deux cités grecques ne souffle mot sur la

14 Ibid

15 Ibid.

16 I. Magnesia, $35\left[\mathrm{n}^{\circ} 46 \mathrm{c} P L G\right]$.

17 A. REHM, Milet, I, $3, \mathrm{n}^{\circ} 37$ [n 56 PLG]

18 J'omets de cette liste la parenté entre les Héracléotes du Latmos et les Étoliens (L. RoBert, BCH, 102 [1978], p. 477-490, repris dans Documents d'Asie mineure, Paris, 1987, p. 173-186 [ $\mathrm{n}^{\circ} 15$ PLG]) A. Giovannini l'englobe sous prétexte que les Héracléotes se considèrent comme les colons des Étoliens. À la suite de L. Robert, j'estime au contraire qu'il s'agit d'une parenté mythique puisque le lien entre les deux peuples remonte à Endymion, père d'Aitôlos, l'éponyme des Étoliens. Je ne tiens pas compte non plus de celle entre Milet et Apollonia du Rhyndacos (A. RenM, Milet, I, 3, n 155 [n 58 PLG]), mais pour des raisons que j'explique ci-dessous, p. 189-190.

19 J. et L. RoBert, Bull. Epigr. (1962), n² 204 [n² 20 PLG].

$20 S E G, \mathrm{XIX}, 468\left[\mathrm{n}^{\circ} 21 P L G\right]$.

21 SEG, XII, $379\left[\mathrm{n}^{\circ} 24\right.$ a $\left.P L G\right]$.

$22 S E G, \mathrm{XII}, 380$ [n 24 b $P L G]$.

23 I. Magnesia, $61\left[\mathrm{n}^{\circ} 46\right.$ a $\left.P L G\right]$.

24 I. Magnesia, 80 ou 81 [n 46 b PLG]. Voir L. RoBert, Opera minora selecta II, p. 1072 , n. 2. 
manière de la justifier. Tandis que je pense qu'elles remontent à des généalogies de l'époque mythique et que je tente, dans mon commentaire, d'identifier le héros mythique ou le dieu à l'origine de la parenté, A. Giovannini, lui, considère que ces liens ont une origine historique en ce sens qu'ils proviennent de relations de colonisation. Le débat pourrait se clore là et chaque lecteur pourrait se forger librement une opinion en choisissant l'une ou l'autre des interprétations. Cependant, il me semble que quelques arguments viennent encore étayer la mienne. Les inscriptions exprimant un lien issu de

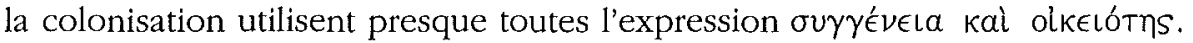
Cette expression est spécifique pour marquer une relation particulière au point que je lui ai consacré un chapitre entier de ma thèse ${ }^{25}$; je constate toutefois qu'A. Giovannini n'en parle pas et pourtant elle est importante. Dans les réponses d'acceptation aux Asclepieia, concours organisé par la cité de Cos, la junctura n'apparaît que dans les décrets de Camarina et de Géla, et, en ce qui concerne les Leucophryena, concours organisé par Magnésie du Méandre $^{26}$, dans celle d'Antioche de Pisidie. Il y a bien plus qu'une coincidence puisqu'il s'agit, les trois fois, d'une réponse d'une colonie à sa métropole ${ }^{27}$.

À Milet, l'expression figure dans deux inscriptions. La première est un traité d'isopolitie conclu par Milet avec Istros ${ }^{28}$, sa colonie, et contenant vrai-

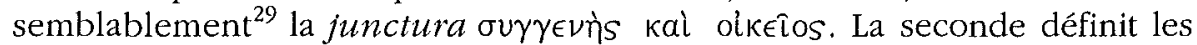
liens entre Milet et la Crète ${ }^{30}$, mais dans un contexte spécialement étroit puisqu'elle traite de l'établissement à Milet de mercenaires crétois et de leur famille.

L'alliance de ces deux mots figure encore dans un décret de Lampsaque ${ }^{31}$ et définit les relations entre la cité et Rome. Le passage qui contient l'expression résume la lettre envoyée à la cité par le commandant de la flotte romaine

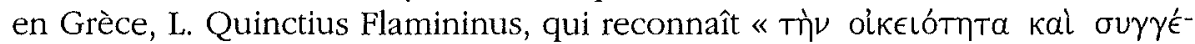
$\nu \in \iota a \nu$ entre les Lampsacéniens et les Romains » (1. 30-31) et exprime par cette expression la bienveillance romaine envers Lampsaque.

26. Pour une analyse plus détaillée des Leucopbryena et des réponses d'acceptation, voir ci-dessous, p. 190-192.

27 Il faut constater que la seconde colonie de Magnésie, Antioche de Perside (I. Magnesia, 61 [ $\mathrm{n}^{\circ} 46$ a $\left.P L G\right]$ ), ne mentionne que la $\sigma u \gamma \gamma \dot{v} v \epsilon \operatorname{co}$. Sur la manière de considérer cette apparente exception, voir ci-dessus, p. 170.

28 L. RoBert, Opera minora selecta I, p. 99-101 [n 54 PLG].

29 Le texte est lacunaire mais permet la restitution de la junctura, dont les deux termes figurent séparément dans le texte, voir n. 54 .

30 A. ReHM, Milet I, $3, \mathrm{n}^{\circ} 37\left[\mathrm{n}^{\circ} 56\right.$ PLG].

$31 S y l l .^{3}, 591\left[n^{\circ} 39 P L G\right]$. 
Une dernière attestation sûre de l'expression figure dans le décret de Larissa en l'honneur d'Hégésipolis, citoyen de Scotoussa ${ }^{32}$. On peut supposer, même si nous n'en avons pas les preuves, que ces deux cités, situées en Pélasgiotide, devaient partager nombre de mythes et de héros qui leur permettaient de se sentir étroitement liées et de s'accorder ces titres.

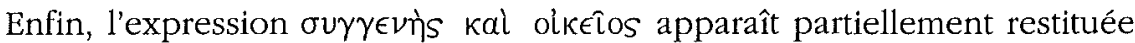
dans le décret argien accordant l'isopolitie aux Aspendiens ${ }^{33}$, là encore dans des circonstances spécialement étroites.

En réunissant les termes exprimant la consanguinité et l'intimité, la junc-

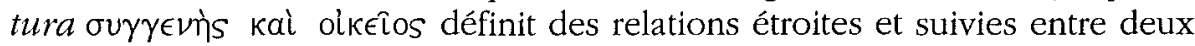
cités. C'est pourquoi, dans les séries de textes semblables, comme les réponses aux Asclepieia de Cos ou aux Leucophryena de Magnésie, l'expression n'est utilisée qu'en rapport avec les colonies de ces cités. Quand la junctura apparaît dans un document isolé, il s'agit toujours de rapports étroits entre deux cités. On peut donc affirmer que l'expression $\sigma u \gamma \gamma \epsilon \nu \eta\rangle \mathrm{s}$ kal

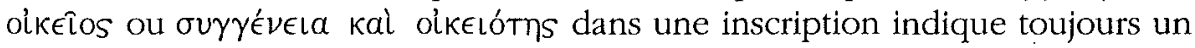
lien privilégié.

Une fois établie cette remarque concernant la forme, je m'intéresserai particulièrement au sens des textes. A. Giovannini prend comme exemple le lien entre les Héracléotes du Latmos et les Étoliens ${ }^{34}$ pour montrer qu'il s'agit là encore d'un cas de parenté dû aux rapports métropole-colonie. S'il est vrai que les Héracléotes sont les colons des Étoliens (1.12-13), la parenté évoquée, elle, remonte bien aux généalogies mythiques par l'entremise d'Endymion, père d'Aitôlos, qui fonda la cité d'Héraclée du Latmos. L. Robert ${ }^{35}$, en effet, a montré que, dans un hymne religieux trouvé à Héraclée et publié par A. Dain en $1933^{36}$, Endymion est explicitement considéré comme le fondateur, кTíotns, d'Héraclée du Latmos. Comme d'autre part Endymion est le père d'Aitôlos, l'éponyme des Étoliens, le lien entre cette Héraclée et les Étoliens est par conséquent très clair. Dans sa vieillesse, Endymion se retira dans le Latmos où il mourut. Comme l'observe L. Robert, « il est facile alors que la ville fondée par Endymion, père d'Aitôlos, ait pu se considérer, au temps de la puissance étolienne, comme la colonie des Étoliens, ămoıкo » avec renvoi aux textes qui attestent, dès le $\mathrm{IV}^{\mathrm{e}}$ siècle av. J.-C., l'existence d'une tradition faisant des Éléens les colons des Étoliens et non l'inverse ${ }^{37}$. Ce texte montre qu'une parenté mythique peut parfois se doubler d'un lien de colonisation, mais il est clair que ce dernier vient, en quelque sorte, légitimer le premier et

\footnotetext{
$32 I G, \mathrm{IX} 2,519$, III [n ${ }^{\circ} 17$ PLG].

33 SEG, XXXIV, $282\left[\mathrm{n}^{\circ} 3 P L G\right]$.

34 Voir n. 18 ,

35 L. ROBERT, op. cit. (n. 18), p. 177-186.

36 A. Dain, Inscriptions grecques du Musée du Louvre, les textes inédits, Paris, 1933, $\mathrm{n}^{\circ} 60, \mathrm{p} .66-73$.

37 L. ROBERT, op.cit. (n. 18), p. 186.
} 
non l'inverse. J'écris bien que ce peut être un lien dû à la colonisation qui vient renforcer et confirmer une parenté fondée sur une tradition mythologique solidement établie. L. Robert ne s'y était pas trompé qui avait identifié Héraclée du Latmos en se fondant sur la généalogie mythologique (élément fort) et non sur la mention de colonie (élément faible).

Le cas d'Alabanda, déjà évoqué ci-dessus à propos de la distinction trop rigide entre les parentés avec des Grecs et des non-Grecs, apporte un argument en faveur de mon interprétation. Voilà une cité qui, comme je l'ai déjà écrit, se déclarait parente de tous les Grecs vers 200 av. J.-C. ${ }^{38}$ alors qu'au III $^{\mathrm{e}}$ siècle apr. J.-C., elle se proclamait cette fois colonie de Sparte ${ }^{39}$. Si l'on en restait là, ce cas ne ferait que confirmer la théorie d'A. Giovannini selon laquelle la parenté entre cités grecques se justifie par un lien, réel ou supposé, dû à des rapports métropole-colonie. Cependant, de même qu'auparavant l'étape intermédiaire permettait de rejeter la théorie trop rigide de la distinction des parentés avec les Grecs ou les non-Grecs, on constate à nouveau que vers la fin du $\mathbf{I I}^{\mathrm{e}}$ siècle av. J.-C., Alabanda se rattachait à la cité eubéenne de Carystos ${ }^{40}$ par l'entremise de la généalogie de Chrysaor, descendant d'Éole par Sisyphe et Glaukos ${ }^{41}$. L. Robert a montré d'ailleurs l'existence à Alabanda de l'anthroponyme très rare $\Sigma\left(\sigma \cup \phi o S^{42}\right.$ qui y atteste l'importance de la généalogie de Chrysaor. Ce nom peu fréquent permet de conclure à la persistance et à la vigueur de la généalogie de Chrysaor à Alabanda. On constate de cette manière que là encore la tradition mythologique s'enracinait au plus profond de l'histoire de la cité, tandis que l'origine lacédémonienne, attestée sur des monnaies du III $^{\mathrm{e}}$ apr. J.-C. ${ }^{43}$, n'avait sa première attestation qu'au II $^{\mathrm{e}}$ siècle de notre ère ${ }^{44}$, lorsque le Spartiate Eudokimos était allé comme juge à Alabanda ${ }^{45}$ car, comme je l'ai déjà releve $e^{46}$, les cités qui avaient besoin de juges se tournaient souvent vers leurs parentes.

Pour conclure, je puis dire que, comme je viens de le montrer, la société grecque antique se définissait surtout par sa rhétorique qui imprégnait chacune de ses actions. Il était, par conséquent, tout à fait normal que les relations de parenté des cités se définissent aussi au moyen de la rhétorique et donc, que les Anciens eussent recours aux généalogies mythiques pour les justifier. Mais un esprit sourcilleux aurait pu encore faire remarquer qu'il ne s'agissait là que d'une simple spéculation. Cependant, la confirmation déci-

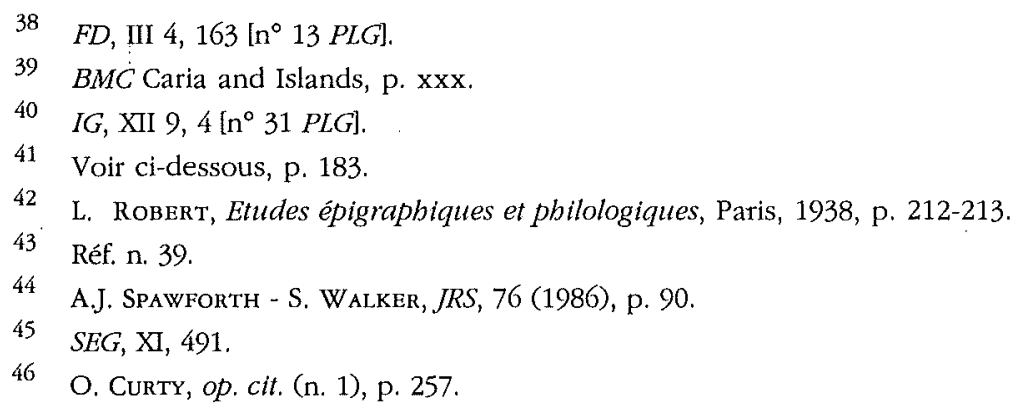


sive a été apportée par la parenté entre les Héracléotes du Latmos et les Étoliens, et celles d'Alabanda. Elles ont confirmé, chacune à sa manière, l'existence d'abord, puis la persistance de traditions mythologiques bien ancrées dans chaque cité. C'est dans ce fonds bien fourni que puisèrent les mythographes pour établir une parenté, ou, plus généralement, pour justifier historiquement tel acte ou telle décision, pour expliquer telle coutume, voire l'origine de tel mot ou encore pour prouver la véracité de tel épisode. Il faut, par conséquent, renoncer à l'hypothèse d'A. Giovannini et accepter le fait que le recours à la mythologie explique les versions parfois contradictoires qui embarrassaient les Anciens eux-mêmes.

\section{L'exemple de Téos}

Toujours dans cette ambiance de «tout mythologique », il paraît intéressant d'examiner un témoignage diplomatique. Quand, en effet, il s'est agi de renouveler son asylie ${ }^{47}$, peut-être vers 160 av. J.-C., la cité de Téos envoya dans les cités crétoises deux ambassadeurs chargés de cette mission en rappelant la parenté entre leur cité et celles qu'ils visitaient. On ignore leur manière de procéder, mais nous possédons deux décrets honorifiques, l'un de la cité de Cnossos, l'autre de Priansos, pour Hérodotos et Ménéclès, les ambassadeurs téiens. Le premier loue Hérodotos d'avoir récité, accompagné de sa cithare, les œuvres des poètes Timothéos de Milet et Polyidos de Selymbria ainsi que celles des anciens poètes locaux ${ }^{48}$. A Priansos ${ }^{49}$, Hérodotos présenta en plus un résumé ${ }^{-50}$ de l'histoire mythologique des dieux et des héros crétois à partir d'œuvres poétiques et historiographiques. Dès lors, il n'est guère difficile de comprendre que la cause de l'éloge pour les ambassadeurs téiens constitue également la base de leur démonstration diplomatique: Hérodotos a profité des recherches effectuées en vue de l'établissement de la parenté et des documents que l'ambassade téienne présentait à l'appui de sa démonstration pour donner un récital dans deux cités au moins ${ }^{51}$.

Cet exemple démontre ainsi à l'évidence la continuité pour les Anciens entre les époques mythique et historique puisque le recours aux événements qui se sont passés dans la première, non seulement permet de justifier la parenté qui se situe dans la seconde, mais est aussi utilisé par les diplomates exactement de la même manière qu'ils traitent les événements quotidiens auxquels ils sont confrontés. Cependant, malgré leur caractère nettement

\footnotetext{
47 Une première demande a été faite à la fin du $\Pi^{\mathrm{e}} \mathrm{s}$. av. J.-C.

48 I. Creticae, I, vin, 11 et A. Chaniotis, op. cit. (n. 10), p. 348-349.

49 I. Creticae, I, xxiv, 1 et A. Chaniotis, ibid.

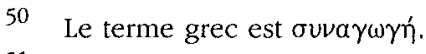

51 Cette production rappelle celle des diplomates mylasiens en Crète également; cf. A. Chaniotis, ZPE, 71 (1988), p. 154-156.
} 
distinct, voire parfois contradictoire, ces deux types ne s'opposent pas car, comme je viens de le montrer, les habitants historiques des cités grecques construisent leur passé à partir de leurs héros épichoriques qui appartiennent à l'époque mythique. On peut encore y ajouter une autre preuve, qui ressortit à la forme : les liens de parenté mythique utilisent un vocabulaire différent de celui de la colonisation ${ }^{52}$. Comme cela a déjà été relevé ${ }^{53}$, les colonies ont, pour parler des relations qu'elles entretiennent avec leur métropole, un vocabulaire spécifique, propre à leur condition. Par conséquent, si une distinction existe bien entre ces deux sortes de vocabulaire, en revanche, je reste persuadé qu'on ne peut pas chercher à tout prix une distinction entre les termes syngeneia et oikeiotès, et il faut admettre, à l'instar de L. Robert, qu'ils sont parfois synonymes ${ }^{54}$.

On peut encore ajouter d'autres cas à l'appui de la démonstration. Ainsi, dans les décisions du décret d'isopolitie entre Pergame et Tégée ${ }^{55}$, trouve-ton le terme oikeiotès, tandis que, dans la formule hortative, il y a syngeneia ${ }^{56}$. Les décrets d'asylie de Téos votés par les cités crétoișes et datant de la fin du Irr $^{\mathrm{e}}$ siècle av. J.-C. comportent la même synonymie entre les deux termes, qui sont utilisés indistinctement l'un pour l'autre. Trois décrets de provenance

52 Voir M. Casevitz, Le vocabulaire de la colonisation en grec ancien, Paris, 1985, qui observe, p. 119, que « $\sigma u \gamma \gamma \in \nu \in \tilde{\text { S }}$ n'est pas sur le même plan que ärtotkol : tandis que

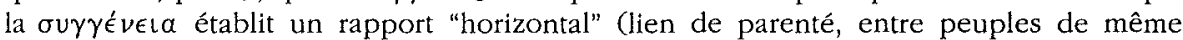
ethnie, Ioniens entre eux, Doriens entre eux etc.), ätoukol note un rapport "vertical" d'un peuple ou d'une cité à un autre peuple, une autre cité, rapport d'origine devenu aussi, vers le $\mathrm{vi}^{\mathrm{e}} \mathrm{s}$., rapport de dépendance, "

O. Curty, op. cit. (n. 1), p. 220-223.

L. Roвert, Opera minora selecta I, p. 100, n. 5: « En général, dans les décrets

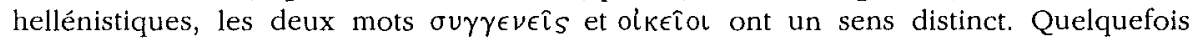
cependant ils sont équivalents; ainsi dans notre texte $\left[n^{\circ} 54 P L G\right]$ et dans le décret de

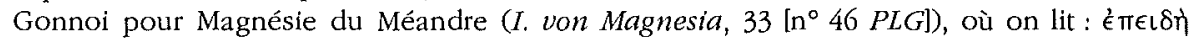

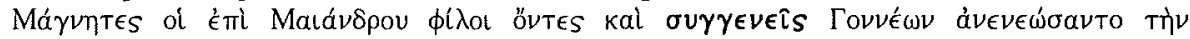

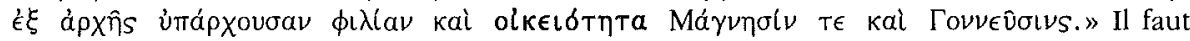
ajouter que j'ai restitué différemment de L. Robert le texte à l'origine de son observation.

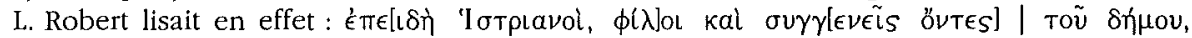

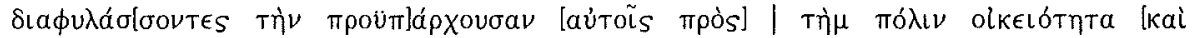

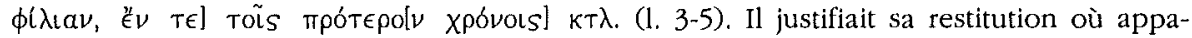

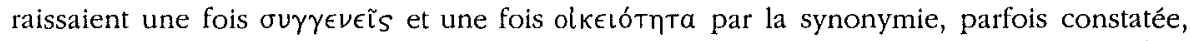
entre les deux termes et cette restitution était à la base de son observation. J'ai préféré

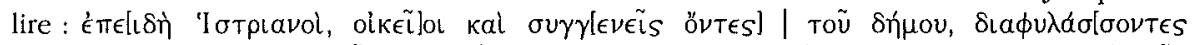

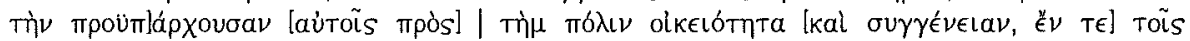

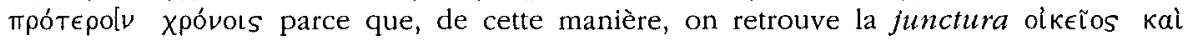

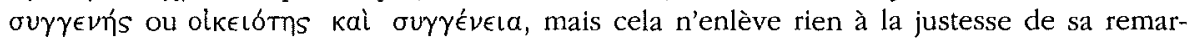
que.

\section{I. Pergamon, I, $156\left[\mathrm{n}^{\circ} 41 \mathrm{PLG}\right]$.}

56 Il me semble que c'est surinterpréter le texte et lui conférer une distinction qu'il ne contient pas que d'écrire comme le fait É. Wris, art. cit. (n. 3), p. 317, n. 31 : « Contrairement à l'oikéiotès, elle (i.e. la syngeneia) n'est pas "fonctionnelle" dans l'accord conclu entre les deux cités : ce n'est qu'un pieux ornement qui vient de surcroît. » 
identique mettent en lumière ce trait. Pour le premier, il s'agit du décret entre Téos et Kydonia ${ }^{57}$ dans lequel les Téiens sont dits $\phi i ́ \lambda o l$ kal $\sigma v \gamma \gamma \epsilon \nu \in \tilde{s}$

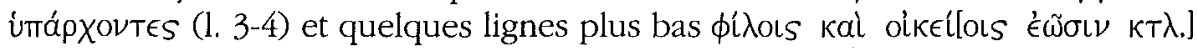
(1. 16). Le deuxième décret, entre Téos et Éleutherne ${ }^{58}$, contient également une attestation de la synonymie des deux termes. Les considérants disent:

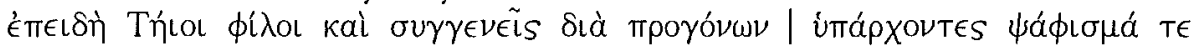

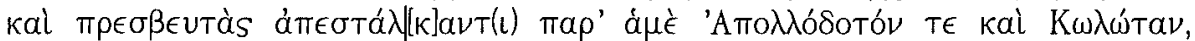

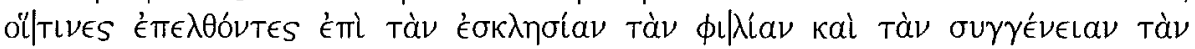

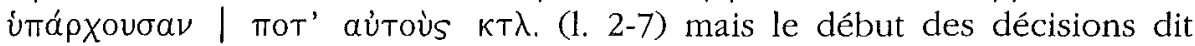

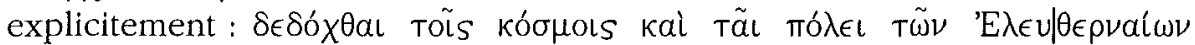

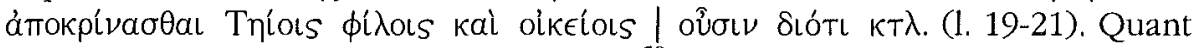
au troisième décret, qui lie Téos à Allaria ${ }^{59}$, il contient encore une confusion plus évidente, si besoin en était, entre syngeneia et oikeiotès. Il dit en effet :

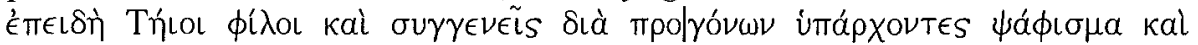

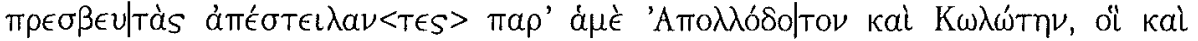

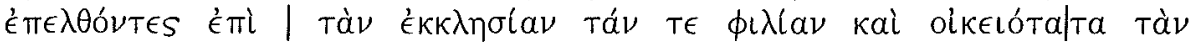

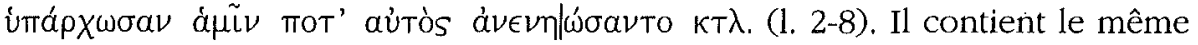

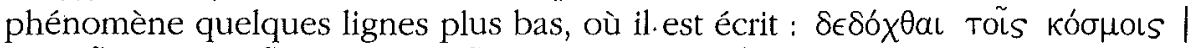

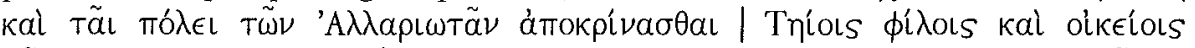

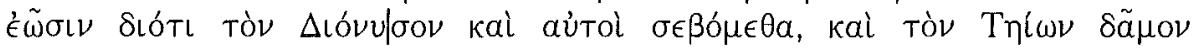

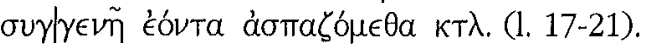

Ces exemples montrent la totale synonymie des termes parce que, si certaines inscriptions opposent entre eux un adjectif et un nom - un esprit

57 I. Creticae, II, x, 2 [n 43 b $P L G]$. É. WILL, art. cit. (n. 3), p. 311, voudrait voir une différence entre syngeneia et oikeiotès puisque le premier terme est défini par l'expres-

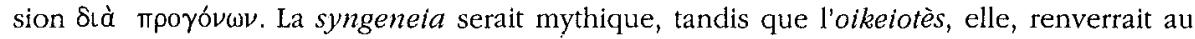
présent. Mais cette démonstration ne tient pas car on peut citer le décret d'Aptère, peutêtre du milieu du II ${ }^{\mathrm{e}}$ s. environ (I. Creticae, II, nı, 2 [n ${ }^{\circ} 43$ j PLG]) qui mentionne la syngeneia sans référence aucune à des progonoi qui puissent être mythiques. On peut encore invoquer le décret d'Hyrtacina, plus tardif, mais concernant le même objet (I. Creticae, II, $\mathrm{xv}, 2\left[\mathrm{n}^{\circ} 43\right.$ o $\left.P L G\right]$ ) avec le même argument. On pourrait, à la rigueur, objecter à cette argumentation que, dans ces deux cas, le terme oikelos est absent des textes. Une réfutation de cet argument est fournie par l'inscription d'une cité crétoise non identifiable, de la même série que la précédente, (SEG, IV, 600 [n 43 p PLG]) où, syngeneia et oikeiotès étant utilisés chacun sans précision, cela empêche formellement de distinguer une nuance entre les deux termes. C'est pourquoi je ne prendrai pas en compte dans mon analyse cette prétendue distinction. Comme cependant ce décret est le seul de sa série à établir la confusion entre les deux termes, il pourrait éventuellement y avoir des raisons autres que la synonymie pour expliquer le fait. Je les ai déjà expliquées dans ma thèse (p. 105). C'est pourquoi, ce témoignage doit être utilisé avec prudence. En outre, É. Will a bien vu que l'expression $\delta$ ¿à $\pi \rho \circ \gamma o ́ v \omega \nu$ fait normalement référence dans les autres inscriptions à l'époque historique et non à la période mythique, comme il est obligé de

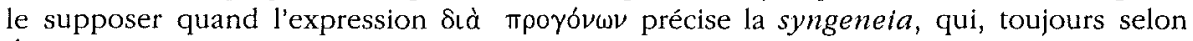
É. Will, renverrait à l'époque mythique. Mais le savant français ne propose aucune solution pour résoudre cette difficulté.

58 I. Creticae, II, XII, 21 [n 43 F PLG],

59 I. Creticae, II, I, $1\left[\mathrm{n}^{\circ} 43 \mathrm{~h} P L G\right]$. 
tatillon, se fondant sur la nature différente des termes opposés, pourrait objecter que la synonymie entre eux n'est pas valable car ils sont de nature différente -, celles qui sont étudiées opposent entre eux soit deux noms, soit deux adjectifs. De cette manière, plus aucune objection n'est possible. On pourrait encore invoquer la convention d'isopolitie entre Milet et Tralles ${ }^{60}$ à l'appui de cette synonymie, mais le traitement qu'elle subit ${ }^{61}$ me poussera ultérieurement à l'analyser en détail. Les cas cités sont malgré tout en nombre suffisant pour corroborer l'affirmation, citée ci-dessus, de L. Robert. Je reviendrai plus tard sur les problèmes liés au vocabulaire, mais je reste pour l'instant aux cas purement épigraphiques.

\section{Réfutation des critiques de J.M. Hall}

Il convenait que ces considérations fussent solidement établies. En vertu de ce que l'on vient de voir du phénomène de la parenté, qui ne traite pas différemment les faits de caractère historique des traditions mythologiques, il est important de voir comment les parentés se constituent avec des récits purement légendaires ou contenant un fond de vérité. La réalité de telles parentés et des discours qui la concernent ne revêt aucune importance du moment que les peuples qui l'invoquent y croient. Écrire alors, comme le fait J.M. Hall, que seuls les Étéocrétois de Praisos et de Polikhna revendiquaient, à l'époque historique, « any continuity with the populations over whom Minos ruled ${ }^{62}$, n'a guère de sens : il n'est pas question, en effet, d'analyser la persistance des liens mythiques à l'époque historique, mais l'usage fait à partir de la période hellénistique de récits légendaires pour justifier des liens entre deux communautés. Sa déformation de l'analyse de la parenté est due, je le suppose, au débordement sur ce phénomène de l'étude de sa propre thèse ${ }^{63}$.

Quant aux relations entre héros qui ne seraient pas consanguines, il cite le cas de la parenté entre Xanthos et Kyténion de Doride ${ }^{64}$. Il écrit « this is an affinal, not a cognative, relationship and ought to have qualified as oikelótms rather than $\sigma v \gamma \gamma \epsilon \dot{v} \epsilon \iota \alpha{ }^{65}$. Pourtant, cette parenté est explicitement justifiée par Létô, grande divinité de Xanthos, dont le fils, Apollon, s'éprit de Coronis, descendante de Dôros, l'éponyme des Doriens, comme son nom l'indique. Asclépios naquit de cette union, qui plus est, en Doride. N'est-ce pas là une preuve tangible de la syngeneia qui existe entre les deux communautés ? Tandis que le terme oikeiotès, que J.M. Hall voudrait utiliser, ne possède, au

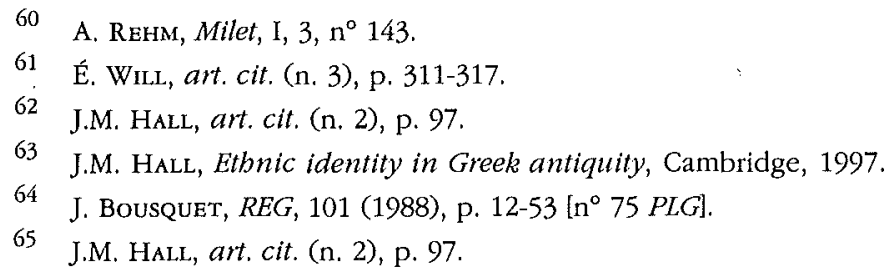


sens premier du mot, que la signification vague d' « intimité des relations » ${ }^{66}$, le terme syngeneia, en revanche, signifie, étymologiquement, « parenté par le sang » et ce mot, au contraire d'oikeiotès, se justifie pleinement dans ce cas.

Pour la parenté entre Argos et Aigée de Cilicie, je renvoie simplement à L. Robert dont la magistrale étude ${ }^{67}$, remplaçant les précédentes, est, bien entendu, citée en tête du commentaire de ma thèse et qui montre que le héros argien Persée, par qui se justifie la parenté entre les deux cités, fit plus que de simplement « se promener » en Cilicie. Il est, en effet, le fondateur de Tarse $^{68}$. De surcroît, le savant français montre que le personnage de Persée était revendiqué par plusieurs cités de Cilicie, comme le prouve leur monnayage $^{69}$. On voit en outre que Persée était particulièrement lié à Aigée car l'inscription mentionne l'apport dans la cité par le héros argien de la statue d'une déesse ancestrale (1. 24-25), mais une lacune malencontreuse ne permet pas de connaître exactement la nature de la parenté. J'avais d'ailleurs déjà relevé ce point en soulignant toutefois que cela n'enlevait rien à l'étroitesse du lien dûment prouvée.

Quant à la parenté entre les Étoliens et les Héracléotes du Latmos, elle n'est pas simplement due au fait qu'Endymion, père d'Aitôlos, éponyme des Étoliens, comme l'indique son nom, se retira dans le Latmos, mais, comme L. Robert l'a bien montré ${ }^{70}$, au fait qu'Endymion est considéré comme le

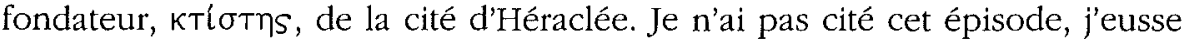
dû le faire. Mais, en revanche, j'ai mentionné à plusieurs reprises l'étude de L. Robert. Un coup d'œil sur cette dernière qui est très aisément accessible aurait permis de comprendre que le retrait d'Endymion dans le Latmos n'était pas chose suffisante pour établir une parenté.

Plus grave est le reproche que m'adresse J.M. Hall à propos de la consanguinité dans la parenté entre Pergame, Pitanè et Mytilène ${ }^{71}$. Il nie, en effet, que les deux cités en litige, Pitanè et Mytilène, aient pu être liées à Pergame, qui réglait leur différend, grâce aux Amazones. Si Pergame fut choisie comme arbitre par l'une et l'autre des cités en litige, il semble relever du pur bon sens que Pergame dut être liée de façon égale à Pitanè et Mytilène pour éviter d'être accusée de partialité en faveur de l'une des cités. Or, pour ce faire, les mythographes trouvèrent la figure d'Andromaque qui s'imposait idéalement. Celle-ci était, d'une part, dans de nombreux récits, la veuve d'Hector et la

66 Sur la signification de ces termes contestée par É. WILl, art. cit. (n. 3), p. 301-311, voir ci-dessous, p. 185-186.

67 L. ROBERT, op. cit. (n. 18), p. 46-90 (= BCH, 101 [1977], p. 88-132). Il faut y ajouter l'article de P. Chuvin, Apollon au trident et les dieux de Tarse, in JS (1981), p. 305-326.

68 L. ROBERT, op. cit. (n. 18), p. ex. p. 57, 59, 71.

69 L. ROBERT, op. cit. (n. 18), p. 74-75.

70 L. Rober't, op. cit. (n. 18), p. 185 (= BCH, 102 [1978], p. 489).

71 I. Pergamon, I, 245 pour la « base " du texte, mais de nombreuses modifications sont apparues depuis lors [ $\mathrm{n}^{\circ} 40$ PLG]. 
mère de Pergamos, l'éponyme de la cité de Pergame ${ }^{72}$. D'autre part, Andromaque était le nom d'une Amazone attestée dans les sources ${ }^{73}$, contrairement à ce que prétend J.M. Hall ${ }^{74}$. Or, les cités de Pitanè et de Mytilène passaient, dans une tradition transmise par Diodore de Sicile ${ }^{75}$, pour avoir été fondées par une Amazone du nom de Myrina. Même si l'Andromaque, mère de Pergamos, est différente de l'Andromaque Amazone, il est de saine méthode, en vertu de ce que j'ai démontré dans ma thèse à propos des liens de parenté établis par l'homonymie de deux héros ${ }^{76}$, d'identifier les deux Andromaque. De cette manière, les deux Amazones, Myrina et Andromaque, permettaient d'établir une parenté entre les trois cités de Pitanè, Mytilène et Pergame. Le lien par l'intermédiaire de ces Amazones est donc réel et non hypothétique comme voudrait le faire croire J.M. Hall.

La parenté entre Mégalèpolis et Magnésie du Méandre ${ }^{77}$ ressortit au même type d'explications que le cas précédent. La parenté dont on se targue tout au long de l'inscription unit Magnésie du Méandre et Mégalèpolis par l'entremise des généalogies des héros de chaque cité, celle d'Éole et de son fils Magnès pour Magnésie du Méandre, celle d'Arcas, éponyme de l'Arcadie, pour Mégalèpolis et plus généralement pour la Ligue arcadienne ${ }^{78}$. Les descendances de ces héros sont bien établies. Éole eut, selon les mythographes, douze enfants, tous connus. Arcas, quant à lui, eut quatre fils, dont les trois premiers s'appelaient Élatée, Aphidas, Azan. Son quatrième fils, Triphylos, eut, quant à lui, une mère différente, dénommée Laodameia ${ }^{79}$. On n'observe aucun élément commun entre les deux généalogies qui eût permis de les attacher l'une à l'autre et, de cette manière, de trouver dans la mythologie un lien qui justifie le titre de parents que s'octroyaient les deux cités. Rien, dans l'inscription, n'indique la manière dont procédèrent les mytho-

72 Pausanias, I, 11, 1-2 et Servius Auctus, Ecl., VI, 72; $c f$. W. Göber, art. Pergamos, in $R E$, XIX 1 (1937), col. 691-692. Ce héros Pergamos, dont on retrouve la trace sur le mon-

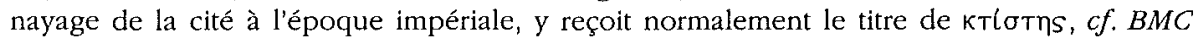
Mysia, p. 136, no 224-226H, pl. XXVIII, 1.

73 Par ex. Schol. in Homerum, Il., III, 189, ed. W. Dindorf, Leipzig, 1877. D'ailleurs, la veuve d'Hector, dont le nom a une connotation guerrière, pourrait être une Amazone à l'origine. Sur Andromaque, voir entre autres P. WATHELET, Dictionnaire des Troyens de

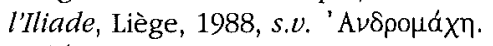

74 J.M. Hall, art. cit (n. 2), p. 97, qui écrit : « a homonymous (but completely unrelated) Amazon. "

75 DIODORE DE SicILe, III, 55, 6-7.

76 O. CuRTy, op.cit. (n. 1), p. 245-247.

77 I. Magnesia, 38 [n $46 \mathrm{~d} P L G]$.

78 Sur l'importance d'Arcas dans les mythes fondateurs de Mégalèpolis et la Ligue arcadienne, voir les remarques M. JOST, Cultes et sanctuaires d'Arcadie, Paris, 1985, p. 448-449. L'inscription de Mégalèpolis à Magnésie est d'ailleurs suivie de la liste des autres cités arcadiennes ayant accepté les Leucopbryena.

79 Telle est la généalogie donnée par la base des Arcadiens à Delphes, $F D$, III 1,3 ainsi que par Pausanias, $\mathrm{X}, 9,5$, qui la décrit. 
graphes pour établir un lien entre les généalogies d'Éole et d'Arcas, mais le cas précédent indique la voie à suivre. Nous avons vu, en effet, que dans des cas semblables, les mythographes confondaient deux héros mythologiques synonymes et, au moyen de ce subterfuge, arrivaient à établir un lien entre deux généalogies autrement irréconciliables. C'est ce qui s'est probablement passé à propos de la parenté entre les cités de Pergame, Pitanè et Mytilène : pour trouver un lien mythologique entre les trois cités, les mythographes ont dû assimiler à l'Andromaque, veuve d'Hector et mère de Pergamos, éponyme de Pergame, l'Amazone du même nom qui permettait un lien avec l'Amazone Myrina, fondatrice de Pitanè et de Mytilène.

Si nous en revenons à notre inscription, force est de constater que seul un tel procédé permet d'expliquer une parenté entre Magnésie du Méandre et Mégalèpolis ou, d'une manière plus générale, la Ligue arcadienne. Dans la mythologie thessalienne, à laquelle se rattachent Éole et son fils Magnès, éponyme du pays de Magnésie, figure un certain Élatée, différent de son homonyme, le fils d'Arcas. Cet Élatée thessalien est un seigneur de la race des Lapithes qui a pour fils Caenée ${ }^{80}$ et l'Argonaute Polyphemos ${ }^{81}$. Or, Magnès et ses fils sont aussi de la race des Lapithes ${ }^{82}$. En identifiant l'Élatée thessalien, Lapithe comme Magnès, à l'Élatée arcadien, l'un des fils d'Arcas, les mythographes anciens trouvaient aisément un lien pour établir la parenté entre, d'une part Magnès, fils d'Éole, éponyme de la Magnésie et, d'autre part, Arcas, éponyme de l'Arcadie. Une confirmation de cette manière de procéder semble se retrouver dans la confusion, relevée à plusieurs reprises par les savants modernes, entre le héros thessalien et le héros arcadien ${ }^{83}$. Nous trouvons d'ailleurs chez Apollodore la trace du mélange des deux généalogies, puisque le mythographe donne comme frère à Caenée, fils de l'Élatée thessalien, Ischys, fils de l'Élatée arcadien ${ }^{84}$. Si aucune indication ne permet d'affirmer que les théores magnètes ont établi leur parenté avec Mégalèpolis

Schol. in Homerum, Il., I, 264

81 Scbol. in Apollonium Rbodium I, 40

82 Cf. W.H. Roscher, Lapithen, RLM, qui recense tous les Lapithes mythiques.

83 A. Kiessling - U. von Wilamowitz-MoellendorfF, Isyllos von Epidautos, Berlin, 1886, p. 59-60; O. WASER, art. Elatos (4), in RE, V 2 (1905), col. 2241, « Die Namen Elatos und Ischys verbinden Thessalien mit Arkadien, und wahrscheinlich ist der Arkader E. identisch mit Lapithenfürst von Larisa » avec renvoi à Wilamowitz-Moellendorff; P. GRIMAL, Dictionnaire de la mytbologie grecque et romaine, Paris, $1982^{7}$, s.v. Elatos, « comme beaucoup de héros arcadiens, Élatos a un doublet thessalien dont il se distingue assez mal. »; B. Helly, Gonnoi. I: la cité et son bistoire, Amsterdam, 1973, p. 65-66, observe à propos de la confusion entre l'Arcadien Gouneus et le héros thessalien homonyme: "l'homonymie des deux personnages montre qu'il existait des rapports entre la Thessalie et l'Arcadie, dans les traditions mythologiques. »

84 APOLLODORE, III, 10, 3. 
par l'entremise de ce héros, nul doute en tout cas qu'ils durent recourir à des procédés identiques ${ }^{85}$.

À propos de la parenté de Carystos avec Alabanda ${ }^{86}$, jugée problématique $^{87}$, l'inscription nous renvoie explicitement à la mythologie puisque le texte mentionne « les liens de parentés entre [leurs] deux cités, qui remontent

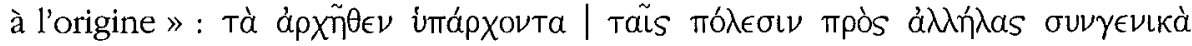
Síkaıa (1. 7-8). La cité d'Alabanda est étroitement liée à la généalogie de Chrysaor, descendant d'Éole par Sisyphe et son fils Glaukos, comme je l'ai déjà démontré dans ma thèse ${ }^{88}$ et rappelé ci-dessus ${ }^{89}$. Or, la cité de Carystos a été fondée par un héros éponyme, fils du centaure Chiron. On ne connaît pas, là non plus, la manière de procéder des mythographes pour établir une parenté mythique entre Carystos et Alabanda, mais plusieurs possibilités s'offraient à eux. Soit ils insistaient sur le lieu de résidence de Chiron, père de Carystos. Chiron avait vécu en Thessalie sur le territoire de Magnésie, cité fondée par Magnès, fils d'Éole et frère de Sisyphe. De cette manière, les mythographes pouvaient relier les deux généalogies. Encore fallait-il qu'ils ajoutent un lien plus étroit car un simple lieu de résidence ne suffisait pas pour établir une parenté. Soit ils partaient des fils de Magnès, Eionée et Eurymonos, petits-fils d'Éole et neveux de Sisyphe, liés l'un et l'autre aux centaures et, par conséquent, à Chiron. Cette solution permettait aussi de rattacher les deux généalogies. Peu importe finalement de savoir pour quelle solution les mythographes ont opté; l'essentiel réside dans le fait que des liens remontant à des épisodes mythiques ont servi à établir une parenté entre Carystos et Alabanda.

Sur la notion même de la syngeneia, J.M. Hall me reproche de ne m'être intéressé qu'à la période hellénistique en négligeant de remonter jusqu'au v $\mathrm{v}^{\mathrm{e}}$ siècle av. J.-C. Je m'en suis cependant aussi occupé indépendamment de ma thèse ${ }^{90}$ et ai relevé que, chez Thucydide, « la $\xi v \gamma \gamma \epsilon ́ v \epsilon ı$ désigne l'appartenance commune à la branche dorienne ou ionienne : sont $\xi \cup \gamma \gamma \in \nu \in \tilde{i}$ s exclusivement les cités doriennes entre elles ou les cités ioniennes entre elles également. On le voit dans de nombreux exemples ${ }^{91}$. Ce trait marque une différence par rapport à la parenté de l'époque hellénistique: il souligne l'importance des liens ethniques. Certes, plus tard, ces liens seront encore invoqués, mais ils ne revêtiront plus le caractère essentiel qu'ils possèdent au

85 D'autres héros ont pu être invoqués pour justifier cette parenté, $c f$. B. HeLLY, op. cit. (n. 83), p. 66 : « des liens s'établissent entre les héros arcadiens et les Lapithes, par l'intermédiaire de Gouneus, de Phénéos et Laonomé » et p. 67-72,

86

87

88

89

ir p. 173.

90 O. Curty, La notion de la parenté entre cités cbez Tbucydide, in $M H, 51$ (1994), p. 193-197.

91 Cf. par exemple Thucydide, III, 2, 3; III, 86, 2-3; IV, 61, 2; IV, 64, 3; V, 104; V, 108. 
$\mathrm{v}^{\mathrm{e}}$ siècle. On justifiera la parenté entre deux cités en recourant à d'autres arguments et l'appartenance à une branche ethnique commune ne sera plus un critère déterminant $~^{92}$. Ce passage prouve à l'évidence que les liens ethniques restent importants à partir du $\mathrm{IV}^{\mathrm{e}}$ siècle av. J.-C. Ce que je prétends $^{93}$ et maintiens, c'est qu'à l'époque hellénistique, à partir de laquelle se manifeste un goût pour les généalogies mythiques, le seul critère ethnique ne suffit plus à établir une parenté entre deux cités. De ce point de vue, les parentés entre les Acarnaniens et Anactorion ${ }^{94}$ ou entre Larissa et Scotoussa ${ }^{95}$, qui datent respectivement de la fin du $\mathrm{III}^{\mathrm{e}}$ et du $\mathrm{II}^{\mathrm{e}}$ siècle av J.-C., s'expliquent très probablement de cette manière. Bien que, là encore, aucune généalogie mythique ne soit mentionnée, il n'est pas trop téméraire de raisonner par analogie. Il est clair que les cités qui se targuaient d'être parentes appartenaient au même groupe ethnique. Mais on vient de voir qu'à partir de l'époque hellénistique, ce critère n'était plus suffisant; il fallait encore que les généalogies mythiques de chaque cité fussent liées entre elles d'une façon ou d'une autre. Comment, dans ce cas, ignorer, même si cela est tacite, que les parentés reposent sur un lien plus profond que simplement l'« ethnic affinity »?

Quant à Cos et Cnide ${ }^{96}$ ou Priène et Érythrées ${ }^{97}$, dont les attestations de parenté sont à peu près contemporaines puisqu'on les place environ dans la première moitié du $\mathrm{II}^{\mathrm{e}}$ siècle av. J.-C., elles ne contiennent, elles non plus, aucune allusion à une quelconque généalogie mythique, mais, du moins, peut-on facilement rattacher l'un à l'autre les systèmes légendaires par l'intermédiaire d'un ou de plusieurs héros mythologiques. Vu la manière de procéder des Anciens, dont les parentés reposent toujours sur des éléments qu'eux-mêmes jugeaient concrets, mais qu'aujourd'hui nous estimons légendaires, il semble licite, même si de plus amples renseignements font défaut, de supposer que des contacts entre cités concernées étaient facilement noués par l'intermédiaire de généalogies mythiques.

Si J.M. Hall me reproche - je crois avoir montré que c'était à tort - de ne pas m'être intéressé au phénomène de la parenté avant l'époque hellénistique, il m'impute, de manière symétrique, la faute de faire une analyse gravement erronée du phénomène de la parenté à l'époque romaine. Il prétend en effet que je serais en pleine contradiction en approuvant les observations de $\mathrm{D}$. Musti sur le caractère artificiel des parentés à la basse époque hellénistique et à la période romaine et, en même temps, en affirmant que les parentés gardent toute leur vigueur jusqu'à l'époque d'Hadrien et du Panhellénion.

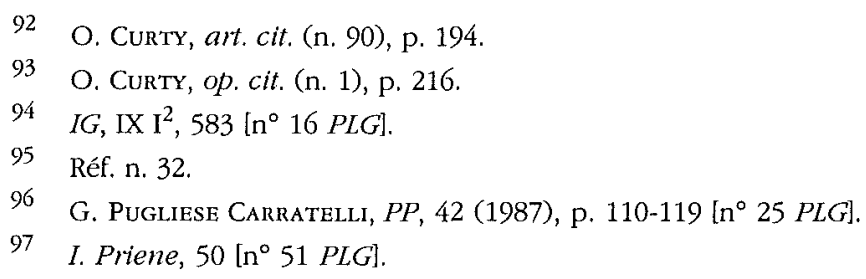


Il n'y a, me semble-t-il, aucune contradiction entre le fait d'affirmer la primauté de liens, surtout ethniques, à la haute époque hellénistique et le fait qu'à la basse époque hellénistique et à la période romaine, ces parentés permettront de rattacher une cité d'Asie Mineure tardivement hellénisée aux cités prestigieuses de l'Hellade. À ce moment-là alors, comme l'avait déjà remarqué $\mathrm{D}$. Musti, l'appartenance commune à une même ethnie ne sera plus un argument important pour établir une parenté; c'est pourquoi elles donneront l'impression d'être artificielles. Cependant, comme je l'ai observé, les parentés, durant toute l'Antiquité, possédèrent une vigueur jamais démentie et eurent toujours besoin d'être justifiées; elles ne purent jamais être accordées par hasard. Comme cela vient d'être dit, leur caractère artificiel - mais pas aléatoire - provient du fait que, si les parentés durent toujours être prouvées, deux cités parentes ne furent plus nécessairement obligées d'appartenir à un même groupe ethnique.

Pour prouver l'évolution durant l'époque hellénistique de la parenté entre cités qui, loin de rester une notion établie une fois pour toutes, se transforma durant cette période, un seul exemple suffira. Ce cas montrera que le lien entre deux cités, par l'intermédiaire d'un héros mythique, tel qu'il se définissait à la haute époque hellénistique, n'était plus de mise à l'époque romaine. Il s'agissait alors, pour une cité quelconque, de se rattacher à l'une des prestigieuses cités de la Grèce. Le cas d'Alabanda est, une fois de plus, très révélateur.à ce sujet. À la fin du III $^{e}$ siècle av. J.-C., la cité qui, dès le règne d'Antiochos II, avait pris le nom d'Antioche des Chrysaoréens ${ }^{98}$, se déclarait parente des Grecs en général ${ }^{99}$. D'origine carienne, Alabanda ne cherchait pas à se rattacher à une cité particulière, mais à se faire admettre par les Grecs en leur sein ${ }^{100}$. Environ un siècle plus tard, la cité, qui avait retrouvé son nom originel, affichait sa parenté avec la cité de Carystos ${ }^{101}$, vraisemblablement par le truchement de Chrysaor, descendant d'Éole, comme je l'ai montré plus haut $^{102}$. Si les deux documents expriment des moments différents dans l'hellénisation de la cité ${ }^{103}$, il n'en reste pas moins que la parenté nouée par l'intermédiaire de Chrysaor et de la généalogie de Sisyphe continue de

98 Sur cette métonomasie ou changement de nom, sa date et sa durée, voir L. RoBer', BCH Suppl. I, 1973, p. 448-466.

$99 F D$, III $4,2,163\left[\mathrm{n}^{\circ} 13\right.$ PLG].

100 Pour un ton semblable, voir les lettres de Ziaèlas de Bithynie (C.B. Welles, Royal Correspondence in the Hellenistic Period, New Haven, 1934, réimpr. Rome, 1966, $\left.\mathrm{n}^{\circ} 25\right)$ et d'un roi du Bosphore (SEG, XII, $370\left[\mathrm{n}^{\circ} 24\right.$ e $\left.P L G\right]$ ) qui, eux aussi, cherchent à se rattacher aux Grecs.

101 Réf. n. 40.

102 Voir ci-dessus, p. 173.

103 Le second document, plus tardif, indique un renforcement de l'hellénisme puisqu'Alabanda se rattache dorénavant à une cité particulière; elle ne se considère plus comme une cité barbare apparentée, mais comme une cité grecque à part entière. 
rappeler les origines cariennes de la cité $e^{104}$. À l'époque romaine, la parenté revendiquée par Alabanda ne relevait plus du même contexte. La cité ne faisait plus désormais référence à une quelconque origine carienne, mais se rattachait directement à Sparte à laquelle elle se prétendait liée comme en témoignent ses monnaies frappées au $\mathrm{III}^{\mathrm{e}}$ siècle apr. J.-C. et portant la légende

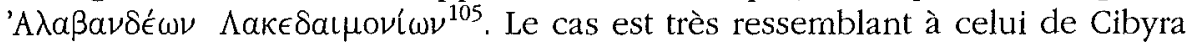
qui, dans une inscription datée du $\mathbf{~ I}^{\mathrm{e}}$ siècle apr. J.-C. et en rapport avec le Panhellénion $^{106}$, se proclama à la fois colonie de Lacédémone et parente d'Athènes ${ }^{107}$.

L'exemple d'Alabanda, loin de révéler une éventuelle contradiction entre la thèse de D. Musti et la mienne, souligne bien, comme j'ai tenté de le démontrer, l'évolution, tout au long de l'époque hellénistique, de la finalité du phénomène des parentés entre cités. Il aboutit, à la période romaine, à un système artificiel, puisqu'une petite cité inconnue peut se rattacher à une glorieuse cité du continent. On observe, cependant, une grande permanence entre la haute époque hellénistique et la période romaine dans la manière de justifier les parentés mythiques.

\section{Analyse des termes syngeneia et oikeiotès}

J'en arrive, comme indiqué, à l'étude plus formelle du vocabulaire. J'aurais, selon $\hat{E}$. Will ${ }^{108}$, analysé de manière tout à fait fausse les valeurs des termes syngeneia et oikeiotès par « manque de rigueur philologique » ${ }^{109}$. Sans volonté de polémiquer, je tiens à répéter ce que j'ai affirmé dans ma thèse et qui me paraît toujours juste malgré les analyses inverses du savant français. Le problème est le suivant : il s'agit de définir les notions de syngeneia et d'oikeiotès ainsi que leurs rapports respectifs. Pour cela, je suis parti non pas des inscriptions contenant ces termes qui définissent des relations entre États, mais des plaidoyers des orateurs attiques où ces termes servent à définir des relations interpersonnelles. Jusque-là, la méthode utilisée ne sou-

104 ÉtIENNE dE BYZance, s.v. 'A $\lambda a ́ \beta a v \delta \alpha$ montre que la cité est attachée par toutes sortes de liens à la Carie.

$105 C f . B M C$ Caria and Islands, p. $\mathrm{xxx}$.

106 Voir en dernier lieu, C.P. JONES, Cbiron, 26 (1996), p. 29-56.

107 IG XIV, 829. On pourrait aussi mettre en rapport le cas de Tabai dont Étienne de Byzance, dans le lemme consacré à cette cité, attribuait l'origine soit à Marsyas, héros spartiate - frère de Kibyras, fondateur de la cité voisine de Cibyra aux origines lacédémoniennes (voir L. et J. Robert, La Carie. II. Le plateau de Tabai et ses environs, Paris, 1954, p. 73-75) -, soit à Tabenos, héros argien (voir L. et J. RoberT, id., p. 88). On constate que, dans l'une ou l'autre possibilité, il s'agit en tout cas de héros originaires de vieilles cités prestigieuses. Ce trait est propre à l'époque du Panhellénion (voir, p. ex., M. SARTRE, L'Orient romain, Paris, 1991, p. 194-196).

108 É. WiLl, art. cit. (n. 3), p. 300-305.

109 Id., p. 300. 
lève pas de problème. Le résultat de l'analyse montre que, dans les relations interpersonnelles, sont syngeneis, comme l'écrit É. Will ${ }^{110}$, « les parents consanguins dans l'ordre patrilinéaire, de la filiation directe au cousinage issu de germains. " Dans l'ordre des relations entre États, la transposition d'un tel concept, la syngeneia, ne pose pas de difficulté. Deux cités, en effet, se disent syngeneis quand elles ont un ancêtre mythologique commun, dieu ou héros ${ }^{111}$.

Le problème survient quand il faut transposer dans le domaine des relations entre États la notion définie par le terme oikeiotès ${ }^{112}$ qui ne laisse pas d'être claire lorsqu'il est question d'individus. : il s'agit d'un lien qui n'est pas de nature consanguine. Pour É. Will, « le rapport entre syngeneia et oikeiotès est un rapport d'extension : les syngeneis, ossature de l'otkos, sont nécessairement des oikeioi, mais la réciproque n'est pas vraie $\mathrm{e}^{113} »$, tandis que moi, j'y vois un rapport d'intensité, «formule dangereusement vague qui [m'] entraîne explicitement sur le terrain de l'affectivité ${ }^{114} »$. Mais le terme oikeiotès, je le répète, possède une valeur moins forte que syngeneia, ce qui n'a aucun rapport avec l'affectivité. De plus, entendre entre syngeneia et oikeiotès un rapport d'intensité n'implique pas qu'une personne syngenès d'une autre, parce qu'elle est son oncle, lui soit plus liée qu'à une troisième "simplement " oikeios, qui ne lui est pas apparentée mais à laquelle des relations fort chaleureuses peuvent la lier. Ce que j'ai dit et que je répète, c'est que les deux mots ne se définissent pas en termes d'affection, mais par plus ou moins d'étroitesse de liens. Je suis d'accord avec É. Will quand il affirme que l'intensité de l'affection n'a rien à voir avec la proximité des relations. Les rapports personnels avec un consanguin sont souvent en effet moins forts qu'avec une personne non apparentée. Le malentendu provient de ma traduction ambiguë du terme oikeiotès par « intimité ». Tandis que je ne lui concède qu'une notion d'intensité, voulant montrer par ce terme une valeur moindre que la « parenté », É. Will a cru - à tort - que j'y voyais une mesure d'affectivité et par conséquent s'en est démarqué, en indiquant que je m'engageais sur une fausse piste. Pour éviter toute équivoque, j'eusse dû

$110 \quad I d .$, p. 301.

111 Si É. Will et A. Giovannini sont unanimes à condamner mon analyse de la syngeneia, en revanche ils divergent quant à l'origine de la parenté entre cités grecques. Pour É. WILL, art. cit. (n. 3), p. 300, - comme pour moi - elle est due à des personnages mythologiques, tandis qu'A. Giovanninr, art. cit. (n. 4), p. 162, l'impute à des liens issus de la colonisation. J'ai déjà démontré plus haut, p. 169-174, ce me semble, l'erreur de cette interprétation.

112 É. WILL, art. cit. (n. 3), p. 301, me reproche de ne jamais avoir mentionné oikia/oikos dans mon analyse de l'oikeiotès entre individus. Outre le fait que l'origine du mot oikeiotès est obvie (de la même manière, je n'ai pas précisé l'étymologie de syngeneia), je cherchais à adapter le terme au contexte international et faire référence à oikia/oikos n'eût pas été à sa place.

113 É. WILL, id, p. 301-302.

114 Id., p. 302. 
choisir un autre mot qui prêtât moins à confusion qu' intimité ». À ma décharge, je dirai que je voulais absolument éviter toute confusion avec un terme de parenté sans voir qu'en choisissant celui d'intimité, j'en créais une autre dans le domaine des sentiments. Ce premier reproche que m'adresse É. Will est par conséquent le résultat d'un malentendu.

En revanche, l'erreur d'analyse que le savant français m'attribue ne trouve pas sa cause dans un malentendu. É. Will en effet dénie catégoriquement au terme oikeiotès la possibilité de signifier, dans un sens technique et en ce qui concerne les relations individuelles, « parenté par alliance ». Là non plus, je n'entends pas réfuter un à un ses arguments, non que je veuille escamoter tout débat, bien au contraire, mais je répéterai, en précisant ma pensée, les arguments en faveur de mon interprétation. Lorsqu'en effet les termes syngeneia et oikeiotès sont utilisés dans un même texte, à quelques lignes de distance, une différence entre eux est perceptible. Je la vois par rapport à la consanguinité. Alors que, dans les relations individuelles, la syngeneia marque la parenté de sang, l'oikeiotès, elle, définit, de manière symétrique, mais opposée, la parenté par alliance. J'aboutis à cette définition non par quelque intuition infondée, mais par une preuve évidente fournie, entre

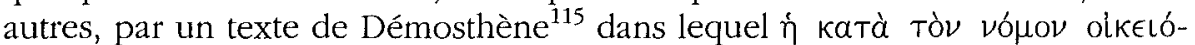

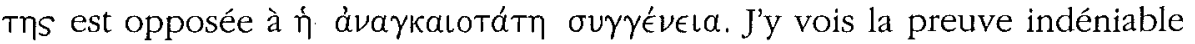
que, dans certains cas, l'oikeiotès, quand elle est utilisée concurremment à la syngeneia, qui a toujours la signification de parenté par le sang, peut signifier parenté par alliance. Je ne vais pas reprendre ici la totalité de ma démonstration dans laquelle j'espère avoir établi les autres significations de l'oikeiotès. Qu'il me suffise de renvoyer le lecteur à ma thèse.

Pour prouver, au contraire, le caractère large et mal défini des oikeioi, É. Will part du passage tiré des paragraphes 67-70 du discours 57 de Démosthène. Il montre que je serais dans l'erreur en donnant ici à oikeios le sens de «parent par alliance». Cependant, le passage que cite É. Will est justement celui que j'invoque pour montrer que parfois, le terme oikeios est utilisé de manière floue et ne se distingue pas de syngenès : il ne signifie alors aucunement « parent par alliance ${ }^{116}$.

É. Will consacre tout un passage ${ }^{117}$ à l'analyse du mot philia, essentiellement dans un discours chez Thucydide. Étant donné que je n'y suis pas pris à partie, je n'ai pas besoin de me justifier. Cependant, je ferai une seule remarque concernant « la valeur neutre » que j'attribue à la philia. É. Will me

115 DÉmosthène, XLIV, 26. É. Will ne cite nulle part ce passage.

116 O. Curty, op. cit. (n. 1), p. 225 : « Considérés séparément, les mots peuvent très bien ne pas posséder de nuance spécifique et être souvent employés indistinctement l'un pour l'autre. Le cas se présente, par exemple, dans un discours de Démosthène où, à

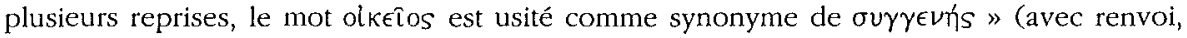
entre autres, au passage incriminé).

117 É. Will, art. cit. (n. 3), p. 305-309. 
reproche ${ }^{118}$ d'octroyer ce qualificatif à la notion de pbilia. Il ne s'agit pas d'un qualificatif donné dans l'absolu, mais au contraire, cet adjectif détermine la philia par rapport à la syngeneia et à l'oikeiotès qui, elles, doivent être justifiées pour pouvoir être octroyées. Je veux dire par l'utilisation du mot neutre entre guillemets que la philia, de son côté, peut être attribuée lorsque les circonstances historiques l'exigent sans que l'existence de liens de parenté doive être établie.

\section{Étude d'un décret de Milet et d'un autre d'Épidamne}

Après avoir voulu - à tort, comme je viens de le montrer - établir, à tout prix, une distinction dans les textes littéraires entre syngeneia et oikeiotès, É. Will veut la retrouver dans les textes épigraphiques. Pour ce faire, il analyse en détail deux inscriptions.

Si je reprends celle de Milet dans laquelle Tralles lui octroie l'isopolitie ${ }^{119}$, chacun peut constater, quelle que soit son analyse, l'évidente synonymie, dans ce texte, entre syngeneia et oikeiotès. Il y a en effet deux décrets, A et B. Le second (B) est un décret de Tralles, qui porte alors le nom de Séleucie. L'autre texte (A), bien que gravé en premier, est en fait postérieur car c'est la réponse milésienne. Les deux textes mentionnent l'un la syngeneia, l'autre l'oikeiotès. Tandis que le décret de Tralles se borne à parler d'oikeiotès et en attribue l'origine au dieu Apollon Didyméen ${ }^{120}$, le décret milésien, quant à lui, fait aussi remonter l'origine des liens au dieu, mais parle de syngeneia ${ }^{121}$. É. Will est très ennuyé car il veut absolument y voir une différence. C'est pourquoi, devant justifier l'utilisation de l'oikeiotès par les Tralliens et celle de la syngeneia par les Milésiens, il est obligé de supposer que les Milésiens substitueraient, à la 1. 9, la syngeneia à l'oikeiotès invoquée par Tralles dans le décret B à la 1. 65. En outre, É. Will est embarrassé par une autre mention, toujours de syngeneia, mais à la 1.4 , cette fois ${ }^{122}$. Il doit justifier sa présence

118 Id., p. 304.

119 A. REHM, Milet I, $3, \mathrm{n}^{\circ} 143\left[\mathrm{n}^{\circ} 55 P L G\right.$ ] avec la nouvelle chronologie de M. WÖrRLE, Cbiron, 18 (1988), p. 428-448.

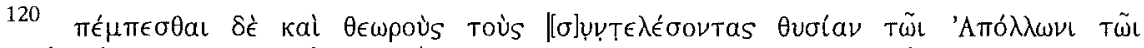

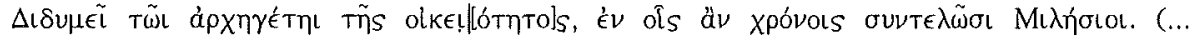
il a plu au Conseil et au peuple) d'envoyer des théores pour accomplir le sacrifice à Apollon Didyméen, fondateur de notre parenté, lorsque les Milésiens les accomplissent (1. 65-66).

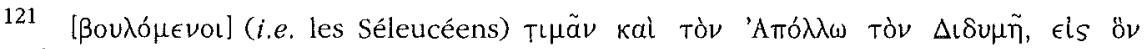

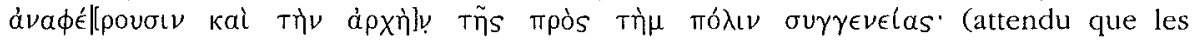
Séleucéens), désireux d'honorer Apollon Didyméen auquel ils font, eux aussi, remonter l'origine de leur parenté avec notre cité (1. 8-9).

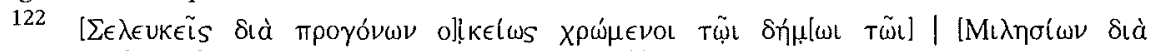

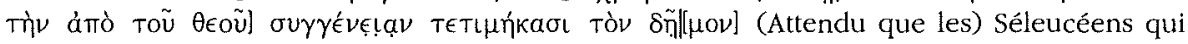
observent par tradition ancestrale une attitude amicale envers le peuple des Milésiens en 
dans les considérants du décret milésien (A) (qui rapporte les propos des Tralliens qui parlent normalement d'oikeiotès). Pour cela, là encore, il est

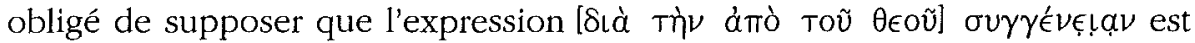
une incise ajoutée par le rédacteur milésien pour préciser l'oikeiotès invoquée par les Tralliens. Ici aussi, l'hypothèse est purement gratuite : rien ne permet de l'étayer. Il y a encore le problème du kai, à la $1.9^{123}$, qui sert à É. Will à prouver le caractère bien distinct de syngeneia et d'oikeiotès ${ }^{124}$. En effet, pour lui, les Tralliens, qui ne mentionnent que l'oikeiotès, auraient été rendus attentifs par les Milésiens au fait que la parenté par le dieu remonterait aussi (kai) à la syngeneia. On aurait ici une preuve certaine de la distinction entre les deux mots puisque dans le décret trallien, Apollon est l'archégète de l'oikeiotès et que, dans le décret milésien, il est aussi à l'origine de la syngeneia. Je ferai deux observations. Premièrement, le kai sur lequel porte le raisonnement est restitué et se trouve dans une lacune et malgré mon « manque de rigueur philologique », je ne me serais pas permis de tirer des conclusions à partir d'une restitution. Deuxièmement, par souci de parallé-

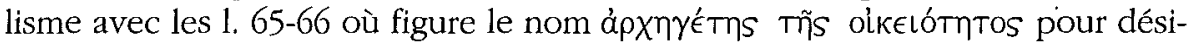

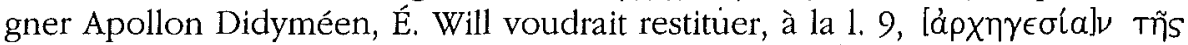

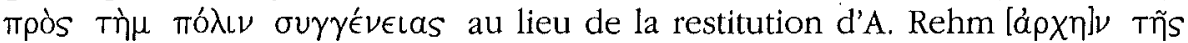

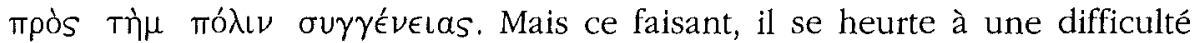
évidente : le manque de place car sa restitution est nettement plus longue que celle d'A. Rehm. Il la résout entre autres en supprimant le kai, fondement même de son analyse, mais maintient cependant son raisonnement. Il s'agit là, me semble-t-il, d'une incohérence flagrante.

Pourquoi les Milésiens ont-ils tant éprouvé le besoin d'invoquer la syngeneia qu'ils l'ont substituée à l'oikeiotès dans un passage et qu'ils l'ont rajoutée dans un autre ? La raison devait être impérieuse pour qu'ils agissent ainsi, mais É. Will avoue son incapacité à trouver une explication ${ }^{125}$. En regard de ce qui vient d'être analysé, je suis conforté dans la démonstration faite dans ma thèse : syngeneia et oikeiotès sont bel et bien synonymes dans ce cas.

Replaçons cet octroi d'isopolitie ${ }^{126}$ dans un contexte plus général. Ainsi Milet distingue-t-elle, en règle générale, ses colonies, des cités parentes. On observe en effet que dans certains décrets, Milet mentionne des liens de pa-

raison de la parenté qui remonte au dieu, ont décerné l'éloge au peuple (des Milésiens) (1. 3-5).

123 Voir n. 121.

124 É. WiLl, art. cit. (n. 3), p. 315-316.

125 Id., p. 316.

126 Je ne suis pas d'accord avec É. Will (id., p. 312), quand il prétend que je n'ai mentionné que par une "fugace allusion » le contenu de l'inscription. Cela voudrait dire pour un lecteur non averti que je n'ai cité qu'incidemment l'objet du décret. Or, j’ai exposé clairement, en début de commentaire, ce dont il s'agissait. 
renté; ainsi avec Istros ${ }^{127}$, la Crète ${ }^{128}, \operatorname{Stratos}^{129}$, Apollonia du Rhyndacos ${ }^{130}$, Thasos $^{131}$, Méthymne et Érésos ${ }^{132}$ ainsi qu'une cité éolienne inconnue ${ }^{133}$. En outre, avec d'autres cités de la région, elle est invitée aux mystères de Panamara en tant que parente de Stratonicée de Carie ${ }^{134}$. Ces cités, qui reçoivent le titre de parentes, ne sont pas colonies milésiennes, hormis Istros et Apollonia du Rhyndacos ${ }^{135}$. En revanche, les traités conclus par Milet avec ses colonies ne comportent aucune mention de la parenté. Dans le décret milésien pour Kios ${ }^{136}$, les rapports entre les deux cités sont clairement établis

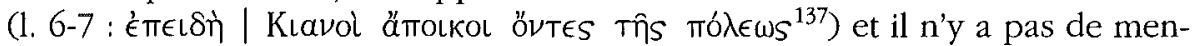
tion de parenté. De même, dans les traités conclus par Milet avec ses colonies un siècle auparavant ${ }^{138}$, ne figure aucun terme de parenté. Le vocabulaire de la colonisation n'y apparaît pas non plus ${ }^{139}$. Donc, aux $\mathrm{IV}^{\mathrm{e}}$ et $\mathrm{III}^{\mathrm{e}}$ siècles av. J.C., lorsque Milet conclut des traités d'isopolitie aussi bien avec ses colonies qu'avec d'autres cités, les titres accordés placent à part les cités parentes, qui ne sont pas assimilées aux colonies ${ }^{140}$. Enfin, une troisième catégorie groupe les décrets milésiens d'isopolitie relatifs à d'autres cités ${ }^{141}$ et se bornant à mentionner des relations d'oikeiotès.

\footnotetext{
$127 \mathrm{~N}^{\circ} 54$ PLG.

128 A. ReHM, Milet I, $3, \mathrm{n}^{\circ} 37\left[\mathrm{n}^{\circ} 56 P L G\right]$.

$129 I G, \mathrm{IX} 1^{2}, 417\left[\mathrm{n}^{\circ} 57 P L G\right]$.

130 A. ReHM, Milet $\mathrm{I}, 3 \mathrm{n}^{\circ} 155\left[\mathrm{n}^{\circ} 58\right.$ PLG].

131 SEG, XXIX, $770\left[\mathrm{n}^{\circ} 59\right.$ PLG].

132 A. REHM, Milet I, 3, $\mathrm{n}^{\circ} 152$ A-B-C [ $\mathrm{n}^{\circ} 61$ PLG].

133 A. ReHM, Milet I, $3, \mathrm{n}^{\circ} 152$ a $\left[\mathrm{n}^{\circ} 60 P L G\right]$.

$134 S E G, \mathrm{IV}, 260\left[\mathrm{n}^{\circ} 70 \mathrm{~h} P L G\right]$.

135 Sur ces deux exceptions, $c f$. ci-dessous, p. 190.

136 Cf. A. Rehm, Milet I, $3, n^{\circ} 141$, avec les remarques de N. Ehrhardt, Milet und seine Kolonien, Francfort-sur-le-Main, $1988^{2}$, p. 47-48.

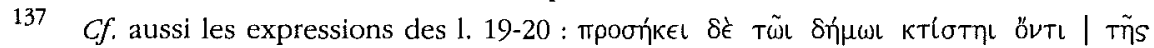

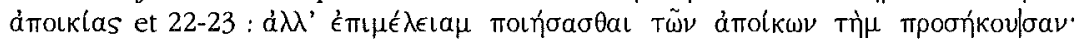

138 Cf. A. R RHM, Milet I, $3 \mathrm{n}^{\circ} 136$ (isopolitie avec Olbia) et $\mathrm{n}^{\circ} 137$ (isopolitie avec Cyzique).

139 Pour A.J. Graham, Colony and Mother-city in Ancient Greece, Manchester, 1964, p. 102-108, ces liens, bien établis, étaient connus de tous et n'avaient pas besoin d'être signalés. De plus, il faut ajouter que, dans le cas de deux cités unies historiquement, comme une métropole et une colonie qui ont gardé le souvenir de leurs liens, les parentés sont nécessairement réelles et éclatent à de nombreuses occasions, lors des cultes par exemple, puisque les deux cités ont les mêmes ancêtres.

140 Ce sont donc deux catégories différentes, au contraire des affirmations de M. Mitsos, $R E G, 59 / 60$ (1946/47), p. 164, qui les confond et parle de relations entre métropole et colonie dans le cas de secours portés par les Stymphaliens à leurs parents, les Élatéens.
}

141 Cf. A. ReHM, Milet I, $3 \mathrm{n}^{\circ} 142$, I. 12 (isopolitie avec Phygela) et $\mathrm{n}^{\circ} 146,1.2,7,60,63$, 81 (isopolitie avec Mylasa). Je laisse de côté le $n^{\circ} 144$, trop fragmentaire pour mon analyse. 
Dans cette répartition, comment expliquer alors la place qu'occupent Istros et Apollonia du Rhyndacos dans le groupe des cités parentes, alors qu'elles devraient se situer dans celui des colonies ? Pour Apollonia, la situation est particulière puisque la cité cherche à se faire reconnaître par Milet comme colonie. Les termes syngeneia et apoikia figurent d'ailleurs côte à côte dans le texte et, pour mettre toutes les chances de leur côté, les Apolloniates ont dû se servir des deux concepts à disposition, sans se soucier de la distinction entre les termes. C'est ce qui explique probablement la place d'Apollonia dans le groupe des cités parentes.

Istros jouit également d'un statut particulier. Au contraire de ce qui se passe pour les cités parentes, les Milésiens ne se contentent pas pour elle de rappeler la parenté, mais mentionnent en plus l'oikeiotès. Le décret pour Istros se distingue de celui pour les autres cités parentes, puisqu'il est le seul, vraisemblablement, à posséder la junctura syngenès kai oikeios et non pas le qualificatif syngenès seul. L'expression syngeneia kai oikeiotès qui sert à marquer un lien particulièrement étroit, est utilisée ici pour décrire les relations entre Milet et Istros, qui sont différentes de celles que Milet entretient avec les cités parentes. Certes, il ne faut pas vouloir chercher dans ces documents une systématisation rigoureuse ni une cohérence stricte qu'ils ne possèdent pas, mais la triple classification des décrets est bien perceptible : la différence entre les colonies, les cités parentes et celles qui ne reçoivent aucun titre: Les textes et le vocabulaire reflètent, confusément parfois, la distinction qu'une cité établit nécessairement entre ses colonies - en quelque sorte chair de sa chair - et les autres cités, lui fussent-elles apparentées par la mythologie. On comprend de cette manière la valeur supérieure du vocabulaire de la colonisation par rapport à celui de la parenté mythique et, dans ce dernier, la valeur moindre d'oikeiotès face à syngeneia.

Un autre document est invoqué à l'appui de la distinction entre les deux termes, c'est le décret d'acceptation des Épidamniens aux Leucophryena ${ }^{142}$. Mais il vaut la peine de le situer dans son contexte. Il fait partie d'un ensemble de documents exposés à Magnésie du Méandre concernant l'institution des fêtes en l'honneur d'Artémis Leucophryène ainsi que la consécration et l'asylie de la cité. Comme en-tête de ce dossier était gravée l'inscription I. Magnesia 16 qui contenait l'exposé des circonstances de l'institution des Leucophryena. Grâce à la réédition de l'inscription par J. Ebert ${ }^{143}$, notre connaissance de l'apparition des Leucopbryena s'est nettement améliorée. D'après ce texte, c'est en 221/0 av. J.-C., sous le stéphanéphorat de Zénodotos, que se plaça une apparition de la déesse Artémis. Les Magnètes

142 I. Magnesia, 46, repris $S y l l .^{3}, 560\left[\mathrm{n}^{\circ} 46 \mathrm{f} P L G\right]$.

143 J. EBERT, Philologus, 126 (1982), p. 198-216; ses nouvelles restitutions sont approuvées et citées par J. et L. RoBert, Bull. Epigr. (1983), n 342; elles sont également reprises dans $S E G$ XXXII, 1147. Ce sont donc ses conclusions que nous reprenons ici, qui rendent caduques les observations de O. KERN, Hermes, 36 (1901), p. 494-498, fondées sur de mauvaises restitutions du texte. 
consultèrent alors l'oracle de Delphes qui leur répondit d'instaurer un concours stéphanite en l'honneur de la déesse. Ils commencèrent par instituer un concours annuel doté d'un prix en argent pour les Grecs d'Asie Mineure ${ }^{144} ;$ treize années plus tard, sous le stéphanéphorat de Moiragoras, en 208/7 av. J.-C., Magnésie du Méandre transforma son concours, selon le vœu de l'oracle, en concours pentétérique, stéphanite, isopythique, musical, gymnique et hippique ${ }^{145}$. L'inscription est muette sur ce retard ${ }^{146}$. Cette même année, plusieurs théories magnètes rendirent visite aux souverains et aux cités grecques pour leur annoncer l'oracle d'Apollon et l'institution de ce nouveau concours organisé pour la première fois la même année ${ }^{147}$. Les réponses d'acceptation de ce concours furent gravées sur les murs du portique ouest de l'agora ${ }^{148}$, probablement peu après 207 av. J.-C. ${ }^{149}$. Les mêmes éléments se retrouvent d'un décret à l'autre: les considérants mentionnent les ambassadeurs magnètes ainsi que l'objet de leur visite - l'institution d'un concours stéphanite pour Artémis, la consécration et l'asylie de leur ville et de son territoire -, tandis que dans les décisions figure l'acceptation de participer au concours et d'observer l'asylie ${ }^{150}$. Le parallélisme est plus étroit

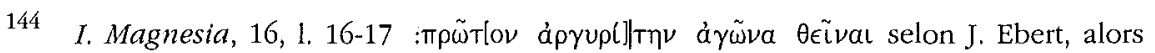
que $O$. Kern restituait $[\sigma \tau \epsilon \phi a \nu i l \mid T \eta \nu$ contresens déjà relevé par L. RoBerT, Opera minora selecta II, p. 776 , n. 1. C'est l'inscription I. Magnesia 15 b, 1. 4-5, qui nous apprend que le concours commença par être annuel.

145 Sur l'apparition et le développement de concours isopythiques ou isolympiques à l'époque hellénistique et impériale, $c f$. les réflexions de L. RoBERT, Opera minora selecta VI, p. $709-719$ (= Actes du VII Congrès international d'épigrapbie grecque et latine, Atbènes 1982 , p. 35-45), et sur la différence entre concours locaux et grands concours internationaux, appelés sacrés par les Grecs, cf. ID., Les inscriptions, in J. DEs Gagniers e.a. (éd.), Laodicée du Lycos. Le nympbée, Québec - Paris, 1969, p. 253.

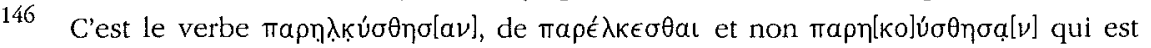
employé ici, avec le sens, selon J. Ebert, de « traîner en longueur ». On ne peut émettre que des hypothèses sur les raisons de cet ajournement, la plus probable selon J. Ebert étant que les Magnètes attendirent qu'une partie au moins des constructions entreprises dans la cité (temple et autel monumental d'Artémis, agora) fût achevée.

147 I. Magnesia, 16, 1. 28-33. Sur la fête des Leucopbryena elle-même, cf. M.P. NiLsson, Griechische Feste von religiöser Bedeutung, Leipzig, 1906, réimpr. Stuttgart 1957, p. 248-251; F. DUNAND, DHA, 4 (1978), p. 201-218 [description fort générale des Leucopbryena].

148 Aucun autre exemplaire de décret d'acceptation n'a été retrouvé ailleurs dans le monde grec, hormis celui de la cité de Delphes, découvert à Delphes même et publié par H. van EfFenterre, BCH, 77 (1953), p. 166-176 (photo dans BCH, 82 [1958], p. 90, fig. 18 ). J. et L. RoBert, Bull. Epigr. (1955), n 122, p. 228-229, ont fait un sort à l'hypothèse de P.M. FrasER, $B C H, 78$ (1954), p. 49-67, qui croyait à tort reconnaître dans des fragments mutilés trouvés à Delphes les restes d'un décret amphictionique acceptant les Leucopbryena.

149 N'ont pas été rendus à cette période 1 . Magnesia 83, décret d'une cité pergaménienne fondée après $208 / 7$ av. J.-C., et 1 . Magnesia, $85,86,87$ qui répondent à une nouvelle annonce des Leucopbryena au milieu du ${ }_{\mathrm{II}^{\mathrm{e}}}{ }$ siècle av. J.-C.; voir O. KeRN, art. cit. (n. 143), p. 515 et L. Robert, Opera minora selecta II, p. 777. 
encore puisque le discours tenu par les théores dans les différentes cités est repris presque dans les mêmes termes chaque fois ${ }^{151}$. On observe la manière identique et répétitive - selon laquelle les théores accomplirent leur mission. Ils commençaient par exposer l'épiphanie de la déesse avec tous les documents utiles à leur démonstration : ainsi rapportaient-ils, avec plus ou moins de détails, les bienfaits accomplis par leurs ancêtres au bénéfice de la cité visitée ${ }^{152}$ ou de la Grèce ${ }^{153}$ et produisaient-ils à l'appui de leurs dires des preuves écrites : décrets, oracles, extraits d'œuvres poétiques ou historiographiques. Ce n'est qu'ensuite qu'ils demandaient d'accepter les Leucophryena ainsi que l'asylie de Magnésie.

C'est dans ce contexte que se place la mention de la parenté; dans certains cas, en effet, les théores magnètes ne se contentaient pas, dans leur préambule, de rappeler aux cités visitées la pbilia ou l'eunoia que Magnésie entretenait avec elles, mais mentionnaient les notions plus prestigieuses d'oikeiotès ou de syngeneia. Dans leurs réponses, les cités reprenaient, lorsqu'il y avait lieu, ces notions valorisantes et, de cette façon, nous sont attestées les différentes parentés de Magnésie. Il devait certainement en exister d'autres, mais deux obstacles nous empêchent de les connaître. Le premier est l'état fragmentaire de nombreux décrets qui rend hypothétique toute restitution de notions comme la syngeneia ou l'oikeiotès et impossible l'attribution à une cité déterminée de certaines inscriptions mentionnant une parenté $^{154}$. Le second obstacle est imputable à l'administration magnète qui décida de ne pas graver toutes les réponses reçues, mais seulement celles de

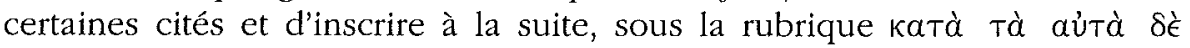
$\dot{\epsilon} \psi \eta \phi i ́ \sigma \alpha v$, les noms des cités voisines qui avaient accédé de manière analogue à sa demande ${ }^{155}$. Il est probable que, dans ces réponses non reprises, des parentés devaient être évoquées, mais l'analyse que j'en fais ne peut spéculer sur de telles hypothèses.

150 Je ne m'arrête pas sur les autres décisions, tels les différents privilèges décernés aux théores.

151 La similitude formelle entre les différents décrets de l'argumentation exposée par les théores atteste la fidélité de la transcription de leurs discours; $c f$. L. RoberT, Opera minora selecta 1,149 , n. 6.

152 Par ex. I. Magnesia, 38, 1. 25-29; 61, 1. 14-19.

153 Par ex. I. Magnesia, 35, 1. 7-10; 46, 1. 8-15; voir A. Chaniotis, op. cit. (n. 10), p. 37 39.

154 C'est le cas de I. Magnesia, $64\left[\mathrm{n}^{\circ} 46 \mathrm{k} P L G\right]$ et, dans une moindre mesure, de $I$. Magnesia, $70\left[\mathrm{n}^{\circ} 46\right.$ j PLG].

155 Ainsi I. Magnesia, 35 (décret de Samè, sur l'île de Céphallénie), qui comprenait à sa suite le nom des trois autres cités de l'île, Palè, Kranies et Pronnes; I. Magnesia, 50 (décret des Pariens), avec la liste des îles voisines. Il est difficile de déceler la raison de ce choix, mais, comme le soutient O. Kern, art. cit. (n. 143), p. 505, il est probable que l'on décida de ne transcrire que les décrets principaux en se bornant à mentionner les moins importants. 
Avant d'examiner l'inscription d'Épidamne, il vaut la peine de dire quelques mots à propos de la fréquente formule oikeios kai philos que j'aurais négligée, sans en voir l'importance selon É. Will. Premièrement, autant dans les réponses d'acceptation aux Leucophryena ${ }^{156}$ que dans le dossier des réponses à la demande d'asylie de $\operatorname{Cos}^{157}$, le couple philos/philia est le plus fréquent, comme É. Will ${ }^{158}$ et moi-même ${ }^{159}$ l'avons relevé et il m'eût été impossible d'en établir un corpus. De plus, cerner une notion aussi complexe que la philia aurait relevé de la gageure. Deuxièmement, mon but, comme il est déjà mentionné dans le titre de ma thèse, était d'étudier une notion à la fois moins courante et plus atypique que l'amitié entre cités : la parenté mythique ${ }^{160}$.

Revenons-en à l'inscription analysée par É. Will. Si les Magnètes sont appelés $\sigma u \gamma \gamma \in \nu \in \tilde{I} S$ kaì $\phi \dot{i} \lambda o t$ (1. 3) par les Épidamniens, en revanche ces derniers sont dits olkeĩol kal $\phi(\lambda$ ol (1. 21) par ceux-là. É. Will, toujours pour distinguer une différence entre ces deux termes, est obligé de supposer, mais sans preuve, que l'oikeiotès fait référence à l'époque historique évoquée par les ambassadeurs magnètes, rappelant les bienfaits accomplis (jusqu'à une époque récente) par leurs ancêtres. L'invocation de la syngeneia, quant à elle, se situerait sur un plan beaucoup moins réel : elle ressortirait au domaine mythologique. Cependant É. Will est sensible à une difficulté : il voit bien qu'en conférant à l'oikeiotès ce caractère concret, on pourrait faire une objection majeure. Si, dans un autre décret du même dossier, les Pariens ${ }^{161}$ octroient aux ambassadeurs magnètes la proxénie et l'évergésie ${ }^{162}$ qui « exprime[nt] sur le plan institutionnel cette vie de relations que je vois à la source de l'oikéiotès ${ }^{163}$, ils ne sont pas dits oikeioi, mais « seulement » pbilo $i^{164}$. Pour résoudre la difficulté et rendre égales dans tous les décrets les différentes notions invoquées par les Magnètes, É. Will est obligé de supposer encore une fois que l'oikeiotès est sous-entendue.

L'analyse aussi bien des orateurs que des inscriptions prouve en conséquence, comme je l'ai relevé, le caractère distinct de la syngeneia et de l'oikeiotès. Mais, avec L. Robert, dont l'observation est reproduite ci-

\footnotetext{
156

I. Magnesia, 16-87.

157 R. Herzog - G. KlafFenbach, Asylieurkunden aus Kos, SDAW 1, 1952.

158 É. Will, art. cit. (n. 3), p. 318.

159 O. Curty, op. cit. (n. 1), p. 236.

160 Pour cette raison, je ne comprends pas la remarque d'É. WiLL, art. cit. (n. 3), p. 317 , n. 34 , qui me reproche de ne pas avoir remarqué la présence d'oikeioi à la 1.21 de I. Magnesia, 46, alors que je ne rapporte que les passages, contenant un membre du

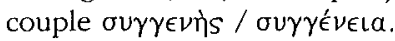
161 I. Magnesia, 50.
162 I. Magnesia, 50, 1. 63-65.
163 É. WILL, art. cit. (n. 3), p. 320.

164 I. Magnesia, 50, 1. 9-10.
} 
dessus $^{165}$, force m'est de constater, en dépit des tentatives d'É. Will, que ces deux termes sont parfois synonymes. C'est en effet la solution la plus simple qui évite toute surinterprétation à laquelle nous conduit immanquablement la volonté de distinguer toujours et partout les deux termes.

D'une manière plus générale, j'observe que mes trois contradicteurs ont ceci de commun qu'ils ont chacun des idées bien arrêtées sur la notion de parenté entre cités. Elles reposent, chaque fois, sur quelques textes seulement. Pour ma part, après avoir constitué un corpus épigraphique, j’ai tiré un certain nombre d'affirmations sans idées préconçues et j'ai abouti à des conclusions différentes des leurs. L'avantage du système que je défends sur les autres, c'est d'expliquer chaque cas avec simplicité et élégance.

Université de Fribourg

Olivier CURTY

Sciences de l'Antiquité

16, rue Pierre-Aeby

$\mathrm{CH}-1700$ Fribourg 